\title{
Dynamic capacitated lot sizing with random demand and dynamic safety stocks
}

\author{
Stefan Helber $^{a}$, Florian Sahling ${ }^{a}$ and Katja Schimmelpfeng ${ }^{b}$ \\ ${ }^{a}$ Department of \\ Production Management \\ Leibniz University Hannover, Germany \\ stefan.helber@prod.uni-hannover.de, ++49 5117625650 \\ ${ }^{b}$ Chair of \\ Accounting and Control \\ Brandenburg University of Technology, Germany
}

February 22, 2011, revised July 18 and November 14, 2011

accepted for publication in Operations Research Spectrum

\begin{abstract}
We present a stochastic version of the single-level, multi-product dynamic lotsizing problem subject to a capacity constraint. A production schedule has to be determined for random demand so that expected costs are minimized and a constraint based on a new backlog-oriented $\delta$-service-level measure is met. This leads to a non-linear model that is approximated by two different linear models. In the first approximation, a scenario approach based on random samples is used. In the second approximation model, the expected values of physical inventory and back$\log$ as functions of the cumulated production are approximated by piecewise linear functions. Both models can be solved to determine efficient, robust and stable production schedules in the presence of uncertain and dynamic demand. They lead to dynamic safety stocks that are endogenously coordinated with the production quantities. A numerical analysis based on a set of (artificial) problem instances is used to evaluate the relative performance of the two different approximation approaches. We furthermore show under which conditions precise demand forecasts are particularly useful from a production-scheduling perspective.
\end{abstract}

Keywords: Stochastic demand, lot-sizing, service level, dynamic safety stocks

Acknowledgement: The authors thank the referees and the editor for their very helpful comments. 


\section{Introduction}

In make-to-stock production environments, production planners often face an uncertain dynamic demand when deciding about the timing and sizing of production quantities. These decisions are often made for a finite planning horizon divided into discrete time periods, e.g., days or weeks. One principle approach to deal with this demand uncertainty is to determine and/or adjust planned production quantities whenever demand for the current period has actually been realized, i.e., the demand is known with certainty. The drawback of this approach is that it can induce substantial system nervousness and, in multi-level supply chains, lead to the notorious bullwhip effect. The other principle approach is to determine dynamic demand forecasts and to establish a production plan that is fixed or "frozen" for the next periods. This schedule is then executed irrespective of the demand realizations for those next periods. While the latter approach leads to more stable planning and production systems, it typically requires safety stocks in order to meet an uncertain customer demand. If demand is uncertain and dynamic, costminimizing planned safety stocks must be dynamic as well. If multiple different product types compete for scarce production capacity, their production quantities and safety stocks need to be coordinated. Furthermore, if setup times and/or setup costs lead to lot sizes covering the demand for multiple consecutive periods, these production lots substantially reduce the need to hold specific safety stocks. Both in the academic literature and in current management practice, these problems are addressed in an often inconsistent and unsatisfactory manner, see Fleischmann (2003). We hence treat these problems jointly and consistently in the context of a proposed Stochastic Capacitated Lot-Sizing Problem (SCLSP), which is a stochastic generalization of the established deterministic Capacitated Lot-Sizing Problem (CLSP).

In this SCLSP, we assume that unmet demand can be back-ordered and that the management uses a constraint on the resulting expected backlog, i.e., the cumulated open (or yet unmet) back-orders, to control the service that is offered to the customers. To this end, the newly developed $\delta$-service-level measure is introduced and used. As both the expected physical inventory and the backlog in the SCLSP are non-linear functions of the production quantities, a non-linear model formulation is developed first. Since we are not aware of a way to solve this first model directly, we further introduce two approximations of the SCLSP by numerically tractable mixed-integer linear models. The first approximation is based on a sample of scenarios, each representing a single realization of the demand process. The second approximation operates with piecewise linear approximations of the expected values of physical inventory and backlog for a given cumulated production quantity. Both modeling approaches are solved using a specific Fix-and-Optimize algorithm as introduced in Helber and Sahling (2010) and Sahling (2010).

The outline of the paper is as follows: In section 2 we present the problem in more detail, analyze service-level measures and discuss the related literature. The model variants of the SCLSP are developed in sections 3 to 5. The Fix-and-Optimize algorithm used to solve both approximation models is outlined in section 6 . Section 7 presents numerical results with respect to the accuracy of the approximation models and the relationship between demand forecasts, lot sizes and dynamic planned safety stocks. Section 8 concludes and outlines future research topics. 


\section{Problem statement, service-level measures and rel- evant literature}

\subsection{Capacitated lot sizing subject to dynamic and stochastic demand}

We assume that a single production system or machine is required to produce $K$ different products. This machine has a (regular) capacity $b_{t}$ in each of the $T$ discrete periods of the planning horizon. It can be extended by overtime at a cost oc per time unit. We do not consider the sequence of the products within any period. If a product $k$ is produced during period $t$, i.e., with production quantity $q_{k t}>0$, a setup time $t s_{k} \geq 0$ is required and a setup cost $s c_{k} \geq 0$ occurs. The processing time for a unit of product $k$ is $t b_{k}$. The cost of holding one unit of physical inventory for one period is denoted as $h c_{k}$.

The demand of product $k$ in period $t$ is modeled as a random variable $D_{k t}$ with a given probability distribution, given expected value $\mathrm{E}\left[D_{k t}\right]$ and variance $\operatorname{VAR}\left[D_{k t}\right]$. The demand for the product-period combination $(k, t)$ is assumed to be independent from those for any other combination $(\hat{k}, \hat{t})$ with $k \neq \hat{k}$ and/or $t \neq \hat{t}$. Estimators of $\mathrm{E}\left[D_{k t}\right]$ and variance $\operatorname{VAR}\left[D_{k t}\right]$ are assumed to be provided by a forecasting system. In order to avoid production schedules that systematically create new backlog at the end of the planning horizon, we assume that for each product $k$, the total production $\sum_{t=1}^{T} q_{k t}$ over the entire planning horizon must at least be sufficient to meet the expected total demand $\sum_{t=1}^{T} \mathrm{E}\left[D_{k t}\right]$.

If in any period $t$ the cumulated (random) demand $\sum_{\tau=1}^{t} D_{k \tau}$ of product $k$ exceeds the cumulated (deterministic) production $\sum_{\tau=1}^{t} q_{k \tau}$, the unmet demand is back-ordered and a positive value of the (random) backlog

$$
B L_{k t}=\max \left(0, \sum_{\tau=1}^{t}\left(D_{k \tau}-q_{k \tau}\right)\right)
$$

occurs. The opposite case results in a positive value of the (random) physical inventory:

$$
Y P_{k t}=\max \left(0, \sum_{\tau=1}^{t}\left(q_{k \tau}-D_{k \tau}\right)\right)
$$

Due to the non-linear maximum functions in equations (1) and (2), both the expected physical inventory $\mathrm{E}\left[Y P_{k t}\right]$ and the expected backlog $\mathrm{E}\left[B L_{k t}\right]$ are non-linear functions of the cumulated production in periods 1 to $t$.

Below we develop a new service-level constraint to limit the total expected backlog relative to the maximum possible total expected backlog. The overall objective is to minimize the expected costs due to setups, inventory and overtime.

If the demand was deterministic and we did not allow any backorders, this setting would describe the Capacitated Lot-Sizing problem (CLSP) which has already attracted a great deal of research, in particular as it is extended to multi-level production systems with multiple capacity constraints and/or to systems in which the setup state can be carried over from one period to the next.

In this paper, however, we assume that demand is stochastic. We still aim at a production plan that is capacity feasible. In addition, this production plan has to be robust in the sense that it systematically reflects demand uncertainty and leads to an a 
priori given service level. After the formal presentation of the model we will show that it leads to dynamic safety stocks that are coordinated with the production quantities.

Note that with respect to the lot sizing aspect of the problem, we are working with a socalled "big-bucket" model as we assume in the (S)CLSP that multiple different products can be produced during a single period. We do not consider the sequence within those periods or setup carry-overs at period borders. If one assumes that (relatively stable) demand forecasts are provided for larger time periods, such as weeks, this appears to be a natural approach. However, in principle it would also be possible to formulate stochastic versions of established "small-bucket" models and hence to integrate sequencing decisions.

\subsection{Service-level measures}

In order to limit the (expected) backlog associated with a production plan subject to random demand, one can penalize it in the objective function using a backlog cost parameter. However, as such a parameter reflects essentially opportunity costs and is hence debatable, it is frequently proposed to limit backorders and/or backlog via a constraint on a suitably defined service-level measure. The following three different service-level measures are frequently discussed in the inventory management literature considering random demand, see Tempelmeier (2006, pp. 26-27):

- The $\alpha$-service level represents the probability that a stockout occurs during a (production or procurement) cycle, or during a period in the rare case of a discrete time model. While this measure may be mathematically tractable and hence attractive from the mathematical point of view, it offers no insights as to how severe the stockout event is once it occurs. From a managerial perspective, the usefulness of this service-level measure seems to be limited.

- The $\beta$-service level (or "fill rate") is the fraction of the demand per cycle that is met immediately, i.e., without backlogging. In a stationary environment these cycles are stochastically identical, so that their average length and hence the $\beta$-service-level can be computed. However, if production quantities $q_{k t} \geq 0$ are determined for each product-period-combination $(k, t)$, a $\beta$-service-level constraint per production cycle can only be enforced once the setup epochs are known. This makes it difficult to simultaneously determine production quantities. Furthermore, the $\beta$-service-level does not reflect the waiting time of the customer. Only the size of the backorder within a cycle is relevant, but not the time required to meet the back-ordered demand.

- Finally, the $\gamma$-service level (here for a specific period $t$ ) is defined as

$$
\gamma_{t}=1-\frac{\text { expected backlog in period } t}{\text { expected demand in period } t}
$$

The attractive feature of this measure is that it reflects backlog and hence, to some extent the waiting time of the customers. It can be defined either (as above) for a specific period $t$, or as an average over the entire planning horizon. However, in a particular period the expected demand may be smaller than the expected backlog or even be zero. Therefore this measure can be negative or even undefined (if the expected demand is zero). From a management perspective, the $\gamma$-service level measure does not seem to offer a clear interpretation. 
In the context of dynamic lot sizing, a backlog-oriented measure seems to be desirable that reflects both the size of the backorders and the waiting time of the customers, but other than the $\gamma$-service-level measure, it should be well-defined and have a clear and transparent interpretation. For this reason, we now define the new $\delta$-service-level measure conceptually as follows:

$$
\delta=1-\frac{\text { total expected backlog }}{\text { total maximum expected backlog }} .
$$

The maximum expected backlog in a period equals the expected cumulated demand including that that of the considered period. It occurs if nothing is produced up to that period. Formally, the $\delta$-service level for product $k$ with random demand $D_{k t}$, deterministic production quantity $q_{k t}$, and random backlog $B L_{k t}=\max \left(0 ; \sum_{\tau=1}^{t}\left(D_{k \tau}-q_{k \tau}\right)\right)$ is computed as

$$
\delta_{k t}=1-\frac{\mathrm{E}\left[B L_{k t}\right]}{\sum_{\tau=1}^{t} \mathrm{E}\left[D_{k \tau}\right]}
$$

for a single period $t$ and averaged over $T$ periods as

$$
\delta_{k}=1-\frac{\sum_{t=1}^{T} \mathrm{E}\left[B L_{k t}\right]}{\sum_{t=1}^{T}(T-t+1) \mathrm{E}\left[D_{k t}\right]} .
$$

The average value of the expected backlog of product $k$ is $\frac{1}{T} \sum_{t=1}^{T} \mathrm{E}\left[B L_{k t}\right]$. Furthermore, the average throughput is $\frac{1}{T} \sum_{t=1}^{T} \mathrm{E}\left[D_{k t}\right]$ if we assume that long-run production meets long-run demand. By Little's Law we have for the average expected waiting time $\mathrm{E}\left[W_{k}\right]$ of product $k$

$$
\mathrm{E}\left[W_{k}\right]=\frac{\frac{1}{T} \sum_{t=1}^{T} \mathrm{E}\left[B L_{k t}\right]}{\frac{1}{T} \sum_{t=1}^{T} \mathrm{E}\left[D_{k t}\right]}=\frac{\sum_{t=1}^{T} \mathrm{E}\left[B L_{k t}\right]}{\sum_{t=1}^{T} \mathrm{E}\left[D_{k t}\right]} .
$$

We can use equation (7) to eliminate the expected backlog from (6) to find the equivalent result

$$
\delta_{k}=1-\frac{\sum_{t=1}^{T} \mathrm{E}\left[D_{k t}\right]}{\sum_{t=1}^{T}(T-t+1) \mathrm{E}\left[D_{k t}\right]} \cdot \mathrm{E}\left[W_{k}\right] .
$$

which shows that the $\delta$-service level is a linear function of the average expected waiting time $\mathrm{E}\left[W_{k}\right]$. Note that this definition of the $\delta$-service level of product $k$ has a clear interpretation: It represents the expected percentage of the maximum possible demandweighted waiting time that the customers of product $k$ are protected against. If no demand is back-ordered, no backlog occurs and we obtain $\delta_{k}=1$. If nothing is produced and delivered until period $T$, all the demand is back-ordered and the maximum possible backlog occurs. In this situation we get $\delta_{k}=0$. All other cases lie somewhere within the interval $[0 ; 1]$. Based on this $\delta$-service-level measure, we develop our optimization models in the next sections. Note that it is not possible to transform this $\delta$-service level over the entire planning horizon into a $\beta$-service-level constraint per cycle as used by Tempelmeier and Herpers (2010) since this requires the additional knowledge of the setup pattern to compute their service measure. 


\subsection{Related work}

Numerous publications address dynamic lot-sizing problems under a broad variety of differing assumptions. However, the overwhelming majority of these works assumes a deterministic setting as the recent reviews by Karimi et al. (2003), Jans and Degraeve (2008), Robinson et al. (2009) and Buschkühl et al. (2010) showed.

While the literature on discrete time, dynamic stochastic lot sizing appears to be relatively limited, a broad body of literature deals with inventory policies. These policies determine on the one hand (often stationary) safety stocks and on the other hand production (or order) quantities as demand is realized over time, see, e.g., the textbooks by Tempelmeier (2006), Zipkin (2000) and the discussion in the older review by Sox et al. (1999). A more recent survey of the "Stochastic Economic Lot Scheduling Problem" was given by Winands et al. (2011) who stated that "... the finite production capacity has to be dynamically distributed among the products in order to be reactive to the stochastic demands, processing and setup times..." (Winands et al. (2011, p. 1)). These "dynamic" and "reactive" elements are the defining features of a production policy serving as a general rule how to act in a future and yet uncertain situation. A typical assumption is that demands "... arrive according to stationary and mutually independent stochastic processes..." (Winands et al. (2011, p. 3)). Our work, however, aims at a specific schedule instead of a general policy for the case of both uncertain and non-stationary demand.

Much of the literature on stochastic lot sizing neither considers non-stationary demand distributions, capacity constraints over multiple products nor the attempt to determine production quantities in advance of the demand realization. The resulting inventory policies can easily lead to a nervous planning system and to the notorious bullwhip effect in multi-level systems. In addition, these approaches also do not reflect the popular practice to operate with "frozen" schedules (Zhao et al. (2001)) to achieve at least some degree of planning stability in the presence of dynamic and/or uncertain demand. Their deficiencies have led to some recent publications. Tunc et al. (2011) addressed the cost of using stationary safety stocks in the case of non-stationary demand and Kanet et al. (2010) emphasized the general positive effect of dynamic planned safety stocks. Absi and Kedad-Sidhoum (2009) presented models to determine production quantities for given values of maximum lost sales and minimum safety stock levels, but did not endogenously determine the latter values.

In a seminal paper for the case of dynamic uncertain demand, Bookbinder and Tan (1988) described three fundamental strategies for probabilistic lot-sizing problems subject to a service-level constraint. In the "static uncertainty" approach, both the timing and the size of production quantities (for a single product) are determined in advance of the demand realizations. The other extreme is the "dynamic uncertainty" approach which assumes that production decisions for a period are made when precise period demands are finally known. To strike a compromise between the extremes, Bookbinder and Tan (1988) proposed the "static-dynamic uncertainty" approach in which the production epochs are fixed beforehand, thus yielding a stable setup pattern, but production quantities are only determined once the demand has been realized. Customer service is reflected via the $\alpha$-service level which limits the probability of a stock-out or back-order event.

In this paper, we use an adapted version of the "static uncertainty" approach as a benchmark to evaluate our own schedules. The basic idea of this approach as presented by Bookbinder and Tan is to transform a single-product dynamic lot-sizing problem subject to random demand and an period-specific $\alpha$-service level constraint into a corresponding deterministic dynamic lot-sizing problem of the Wagner-Whitin type by adjusting the 
demand data. In order to determine the adjusted demand data $d_{t}$ for each period $t$, Bookbinder and Tan compute the required cumulated production in periods 1 to $t$ for each period $t$ that yields a desired $\alpha$-service level in period $t$. The adjusted demand $d_{t}$ for the deterministic dynamic lot-sizing problem of the Wagner-Whitin type is then simply this required cumulated production until period $t$ minus the required cumulated production until period $t-1$. They furthermore approximate the expected value of the physical inventory by the expected value of the net inventory position, which can be crude.

Several authors solved stochastic uncapacitated lot sizing problems based on scenario trees. Guan and Miller (2008) presented a dynamic programming approach which determines a solution for the stochastic uncapacitated lot sizing problem in polynomial time. Guan et al. (2006) adapted the well-known valid inequalities proposed by (Pochet and Wolsey 2006, p. 321) and showed that these are also valid for the case of stochastic demand. Based on these results, Di Summa and Wolsey (2008) presented simplified valid inequalities and reformulations. Brandimarte (2006) proposed a model formulation of the stochastic capacitated lot sizing problem that is also based on a scenario tree. The author reformulated this lot sizing problem as a simple-plant location problem and proposed a Fix-and-Relax heuristic.

Tempelmeier and Herpers (2010) have recently argued that it may be desirable to determine both the timing and the size of production orders based on forecasted demand and keep both fixed or "frozen" over the complete planning horizon, i.e., to follow the "static uncertainty approach". This seems to be particularly important if production schedules have to be coordinated over multiple products due to multi-level production processes or capacity restrictions of the production system. Tempelmeier and Herpers (2010) studied such a stochastic version of the CLSP. They assumed in a specific $\beta$ service-level constraint, that during each production cycle only a maximum fraction of the demand of this particular cycle may be back-ordered. In their paper, a modified version of the ABC-heuristic by Maes and van Wassenhove (1986) was used to determine production quantities. In a subsequent paper, Tempelmeier (2011) presented a different solution approach based on column generation to solve the same problem. It significantly outperforms the adapted ABC-heuristic. The research presented in our paper is on the one hand closely related to those presented in Tempelmeier and Herpers (2010) and Tempelmeier (2011). Instead of limiting back-orders via a $\beta$-service-level constraint, we aim at limiting backlog and hence take the customer waiting time into account using the $\delta$-service level. Furthermore, we impose a service-level constraint for the entire planning horizon instead of one for each single production cycle. On the other hand, the most closely

related papers were presented by Martel et al. (1995) and Sox and Muckstadt (1997) who penalized backlog in the objective function via a penalty cost which did not give them a direct control on the backlog and motivated us to work with a backlog-oriented service level measure.

\section{The non-linear stochastic capacitated lot-sizing prob- lem (SCLSP) with a $\delta$-service-level constraint}

Based on the assumptions in section 2.1, the $\delta$-service level introduced in section 2.2, and the notation in Table 1, we now state the SCLSP as follows: 
$\underline{\text { Indices and index sets: }}$

$\mathcal{K} \quad$ set of products $(k \in\{1, \ldots, K\})$

$\mathcal{T} \quad$ set of periods $(t \in\{1, \ldots, T\})$

Deterministic parameters:

available capacity in period $t$

$\delta_{k} \quad$ minimum $\delta$-service level of product $k$

$h c_{k} \quad$ holding cost of product $k$ per unit and period

$M \quad$ big number

$o c \quad$ overtime cost per unit of overtime

$s c_{k} \quad$ setup cost of product $k$

$t b_{k} \quad$ production time per unit of product $k$

$t s_{k} \quad$ setup time of product $k$

Random variables:

$B L_{k t} \quad$ backlog of product $k$ in period $t$

$D_{k t} \quad$ demand of product $k$ in period $t$

$Y_{k t} \quad$ net inventory position of product $k$ at the end of period $t$

$Y P_{k t} \quad$ physical inventory of product $k$ at the end of period $t$

Decision variables:

$o_{t} \quad$ overtime in period $t$

$q_{k t} \quad$ production quantity (lot size) of product $k$ in period $t$

$x_{k t} \quad$ binary setup variable of product $k$ in period $t$

\section{SCLSP Model}

$$
\min Z=\sum_{k \in \mathcal{K}} \sum_{t \in \mathcal{T}} h c_{k} \cdot \mathrm{E}\left[Y P_{k t}\right]+\sum_{k \in \mathcal{K}} \sum_{t \in \mathcal{T}} s c_{k} \cdot x_{k t}+\sum_{t \in \mathcal{T}} o c \cdot o_{t}
$$

subject to:

$$
\begin{array}{ll}
\sum_{k \in \mathcal{K}}\left(t s_{k} \cdot x_{k t}+t b_{k} \cdot q_{k t}\right) \leq b_{t}+o_{t}, & \forall t \\
q_{k t}-M \cdot x_{k t} \leq 0, & \forall k, t \\
Y_{k, t-1}+q_{k t}-Y_{k t}=D_{k t}, & \forall k, t \\
Y P_{k t}=\max \left(0, Y_{k t}\right), & \forall k, t \\
B L_{k t}=\max \left(0,-Y_{k t}\right), & \forall k, t \\
\sum_{t \in \mathcal{T}} q_{k t} \geq \sum_{t \in \mathcal{T}} \mathrm{E}\left[D_{k t}\right], & \forall k \\
\sum_{t \in \mathcal{T}} \mathrm{E}\left[B L_{k t}\right] \leq\left(1-\delta_{k}\right) \sum_{t \in \mathcal{T}}(T-t+1) \mathrm{E}\left[D_{k t}\right], & \\
q_{k t} \geq 0, & \forall k \\
o_{t} \geq 0, & \forall k, t \\
x_{k t} \in\{0,1\}, & \forall t \\
& \forall k, t
\end{array}
$$


The objective (9) is to minimize the total expected costs of physical inventory, setups, and overtime. Constraints (10) guarantee that the capacity needed for setups and production does not exceed the sum of regular and overtime capacity. Constraints (11) enforce setups in production periods. The inventory balance equations (12) relate the (random) end-of-period inventory position $Y_{k t}$ to the random demand, the production quantity and the inventory position from the previous period. In the following equations, (13) and (14), the physical inventory as well as the backlog are determined. Constraints (15) make sure that at least the expected cumulated demand of product $k$ is produced until the last period $T$. Furthermore, constraints (16) ensure that the backlog does not exceed the target $\delta$-service level.

In this context of the SCLSP, we can now formally introduce the concept of a dynamic safety stock $s s_{k t}$ of product $k$ in period $t$. Though the safety stock is not explicitly modeled in the SCLSP, it can be derived from its solution. Assume to this end that an arbitrary solution to the SCLSP is given. This solution does only not specify production quantities $q_{k t}$, but also production cycles, each starting with a setup period and ending with the last period preceding the next setup. We now define the safety stock $s s_{k t}$ of product $k$ in period $t$ as the difference between the cumulated production quantities and the cumulated expected demand served by the lots up to period $t$ :

$$
s s_{k t}=\sum_{\tau=1}^{t} q_{k \tau}-\sum_{\tau=1}^{\min \left(T, \min \left(\theta \mid(\theta \geq t) \wedge\left(x_{k, \theta+1}=1\right)\right)\right)} E\left[D_{k \tau}\right] \quad \forall k, t
$$

Note that the safety stock is constant within a production cycle, but can differ from cycle to cycle. Note furthermore that it can be both positive and negative. In our numerical analysis in section 7 we will use examples to show the impact of different problem parameters on these safety stocks.

Unfortunately, we are not aware of a method available to solve the SCLSP in the non-linear form presented above. For this reason we develop below two different models to approximate the SCLSP. Both can be solved using standard methods for mixed-integer programming.

As a benchmark, we compare the solutions of these approximation models in sections 4 and 5 to those obtained by a modified "static uncertainty approach" as proposed by Bookbinder and Tan (1988), see section 2. Following Bookbinder and Tan (BT), we have to transform the SCLSP into an corresponding deterministic model with specific deterministic demand. To this end, we determine for each product $k$ and period $t$ the necessary cumulated production of product $k$ over periods 1 to $t$ such that a given period-specific $\delta$-service level $(5)$ is met in period $t$. The deterministic demand in the corresponding BT model for a product in period $t$ is then simply this required cumulated production over periods 1 to $t$ minus the required cumulated production over periods 1 to $t-1$ leading to the desired $\delta$-service level in the respective periods. This demand data is then used in a deterministic CLSP. As a benchmark, we hence extend the approach by Bookbinder and Tan by

- replacing the period-specific $\alpha$-service level constraint by a period-specific $\delta$-service level constraint,

- considering a constrained resource and overtime as in constraints (10), and

- adding a constraint (15) on the total production. 
For the resulting schedules we then determine the expected value of the backlog and the physical inventory under uncertain demand and finally the costs. As these schedules meet the required $\delta$-service level for each single period, they also always meet the aggregate service level constraint (16). As a matter of fact, they tend to exceed the aggregate constraint which leads to schedules that are more costly than those that match but do not exceed the aggregate service level constraint. As this benchmark approach was inspired by Bookbinder and Tan, we call it CLSP-BT.

\section{Linear approximation of the SCLSP via a scenario approach}

\subsection{Approximating expected values by sample averages using scenarios}

The basic idea of the first approximation model is to consider a set of demand scenarios $s \in \mathcal{S}$. Each such scenario $s$ is a random trajectory that represents one of several equally likely paths of realizations of the random demand variables $D_{k t}$ for all $k$ and $t$. In order to achieve a robust production plan that meets the required $\delta$-service level, we optimize the production quantities over the entire set of scenarios $s \in \mathcal{S}$. Random variables $D_{k t}, Y_{k t}$, $Y P_{k t}$, and $B L_{k t}$ are therefore replaced by their respective scenario-specific realizations $d_{k t}^{s}, y_{k t}^{s}, y p_{k t}^{s}$, and $b l_{k t}^{s}$ for $s \in \mathcal{S}$. Furthermore, expected values of these random variables in the SCLSP are approximated by sample averages as follows:

$$
\begin{array}{rlrl}
\mathrm{E}\left[D_{k t}\right] & \approx \frac{\sum_{s \in \mathcal{S}} d_{k t}^{s}}{|\mathcal{S}|}, & \forall k, t \\
\mathrm{E}\left[Y P_{k t}\right] \approx \frac{\sum_{s \in \mathcal{S}} y p_{k t}^{s}}{|\mathcal{S}|}, & \forall k, t \\
\mathrm{E}\left[B L_{k t}\right] \approx \frac{\sum_{s \in \mathcal{S}} b l_{k t}^{s}}{|\mathcal{S}|}, & \forall k, t
\end{array}
$$

Other applications of this sample average approximations can be found, e.g., in Bihlmaier et al. (2009), Helber and Henken (2010). A general overview of scenario analysis methods is presented, e.g., in Mißler-Behr (1993), while Freimer et al. (2010) discuss the impact of different sampling methods in linear models using sample average approximations.

\subsection{Formulation of the SCLSP-SCN}

The modified or additional notation of this second model is shown in Table 2. With this notation, the scenario approximation model SCLSP-SCN can be formulated as follows:

SCLSP-SCN Model

$$
\min Z=\sum_{k \in \mathcal{K}} \sum_{t \in \mathcal{T}} h c_{k} \cdot\left(\frac{\sum_{s \in \mathcal{S}} y p_{k t}^{s}}{|\mathcal{S}|}\right)+\sum_{k \in \mathcal{K}} \sum_{t \in \mathcal{T}} s c_{k} \cdot x_{k t}+\sum_{t \in \mathcal{T}} o c \cdot o_{t}
$$

subject to constraints (10), (11), (15), (17), (18), (19) and: 
$\underline{\text { Indices and index sets: }}$

$\mathcal{S} \quad$ set of scenarios $(s \in\{1, \ldots, S\})$

Scenario-specific parameters:

$\overline{d_{k t}^{s}} \quad$ demand of product $k$ in period $t$ and in scenario $s$

Scenario-specific decision variables:

$b l_{k t}^{s} \quad$ backlog of product $k$ in period $t$ and in scenario $s$

$y_{k t}^{s} \quad$ net inventory of product $k$ at the end of period $t$ and in scenario $s$

$y p_{k t}^{s} \quad$ physical inventory of product $k$ at the end of period $t$ and in scenario $s$

$$
\begin{array}{ll}
y_{k, t-1}^{s}+q_{k t}-y_{k t}^{s}=d_{k t}^{s}, & \forall k, t, s \\
y p_{k, t}^{s} \geq y_{k, t}^{s}, & \forall k, t, s \\
b l_{k, t}^{s} \geq-y_{k, t}^{s}, & \forall k, t, s \\
\sum_{t \in \mathcal{T}} \frac{\sum_{s \in \mathcal{S}} b l_{k t}^{s}}{|\mathcal{S}|} \leq\left(1-\delta_{k}\right) \sum_{t \in \mathcal{T}}(T-t+1) \mathrm{E}\left[D_{k t}\right], & \forall k \\
y p_{k t}^{s}, b l_{k t}^{s} \geq 0, & \forall k, t, s
\end{array}
$$

All the constraints of the SCLSP in section 3 that contain random variables $(12$, 13 , and 14) are replaced by $|\mathcal{S}|$ systems of constraints (25)-(27) reflecting independent scenario-specific realizations of the random variables. Instead of the expected value of the physical inventory in the objective function (9) and the expected value of backlog in the $\delta$-service-level restriction (16), we now use sample averages in the objective function (24) and the service-level constraint (28) as explained in section 4.1. Note that in order to minimize costs, both backlog and physical inventory will tend to be minimized. For this reason, it is possible to replace the non-linear maximum function in Equations (13) and (14) by the new constraints (26) and (27).

If the standard deviation of the demand $D_{k t}$ equals 0 for all $k$ and $t$, a single scenario represents the entire knowledge about the demand. As a result we obtain a version of the deterministic CLSP in which backlogs are allowed. These backlogs are restricted using the $\delta$-service-level constraint. If we additionally assume the case of a desired $\delta$-service level of $100 \%$, the SCLSP-SCN reduces to be the well-known CLSP.

Note that this scenario approach offers a substantial degree of flexibility. In particular, it is possible to construct scenarios that consistently model probabilistic dependencies of the demands for different products or periods. This is a potentially very useful feature of this approximation model.

The realizations of the demand data $d_{k t}^{s}$ for product $k$ in period $t$ can be determined independently for each single scenario $s$ based on the distribution of the random variable $D_{k t}$. However, this so-called Simple Random Sampling (SRS) tends to lead to a substantial variance of any function computed based on such a sample. As a variance-reducing technique, Saliby (1990) proposed the so-called Descriptive Sampling (DS) which he showed to work extremely well for the Newsboy problem, a problem related to the SCLSP. Descriptive Sampling requires the ex-ante knowledge of the sample size $|\mathcal{S}|$, which is always exactly known in our context. The basic idea is to deterministically construct a set of 


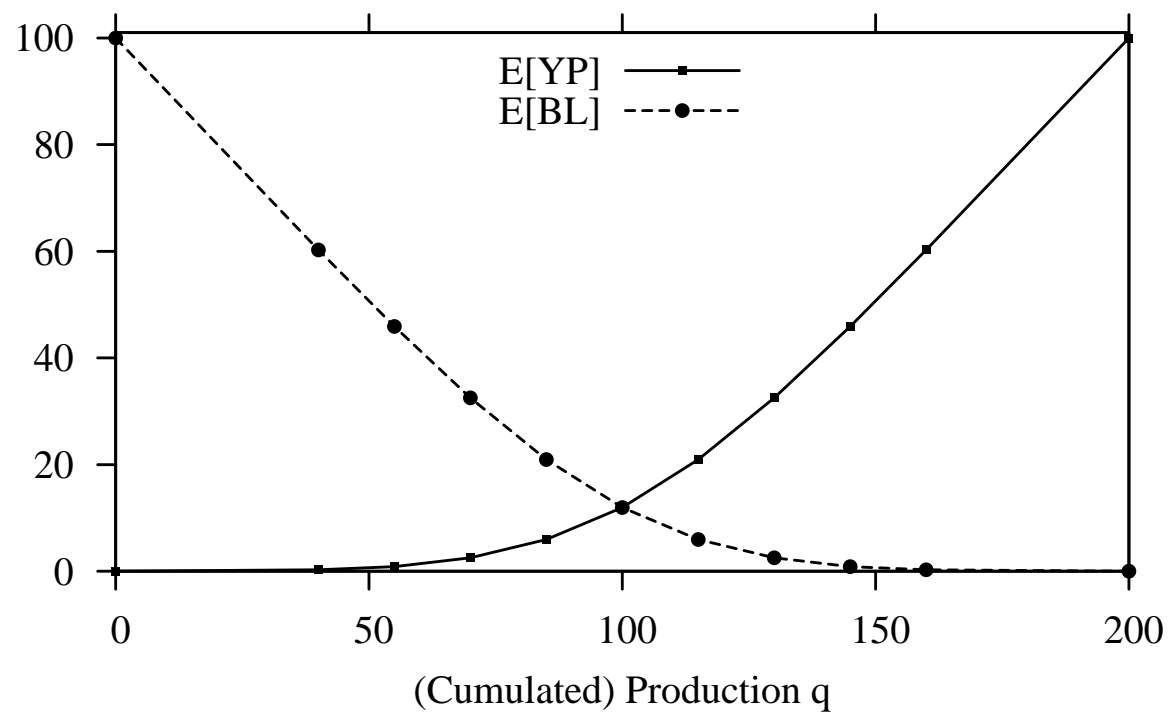

Figure 1: Linearized expected backlog and physical inventory over production

$|\mathcal{S}|$ values $d_{k t}^{s}$ such that their "empirical distribution" follows the underlying theoretical distribution of the random variable $D_{k t}$ as close as possible. Assume, e.g., that for a random variable $Z$ with probability function $F_{Z}(z)$ a set of size 10 is required. Then the set elements are determined by inverting the probability function as $z_{i}=F_{Z}^{-1}((i-0.5) / 10)$ for $i=1, \ldots, 10$ and finally randomly "shuffled" to induce a random sequence. We implemented the SCLSP-SCN with both Simple Random Sampling and Descriptive Sampling to analyze the impact of the different sampling methods.

\section{Piecewise Linear Approximation of the SCLSP}

\subsection{Approximating expected values via piecewise linear func- tions}

It is possible to replace the non-linear functions of expected backlog and physical inventory in the SCLSP by piecewise linear functions. Such a piecewise linearization is shown in Figure 1 for the case of a single period with normally distributed demand, expected demand of 100 and variance of 900. (In the single-period case, the period production equals the cumulated production.) The graph shows that in order to linearize these functions, a point for the minimal (zero) production is required $(q=0)$ and one for the realistic maximum possible production (e.g., $q=200$ ). As we do not have any a priori knowledge about the optimal cumulated production for any given period, the other supporting points of the linearization are concentrated as shown in Figure 1 in the area where the non-linearity of the expected backlog and physical inventory (over the production quantity) is strongest, i.e., around the expected demand, so that the overall deviation from the original non-linear functions is small.

Such a linearization is required for each combination of product $k$ and period $t$ for both the expected physical inventory and the expected backlog as functions of the cumulated production up to period $t$. Let $L$ denote the number of segments of the continuous and piecewise linear functions approximating the non-linear functions of expected inventory or backlog. In the example in Figure 2, the expected inventory is approximated by a piecewise linear function with four segments, i.e., $L=4$. Each such segment $l$ is characterized by 


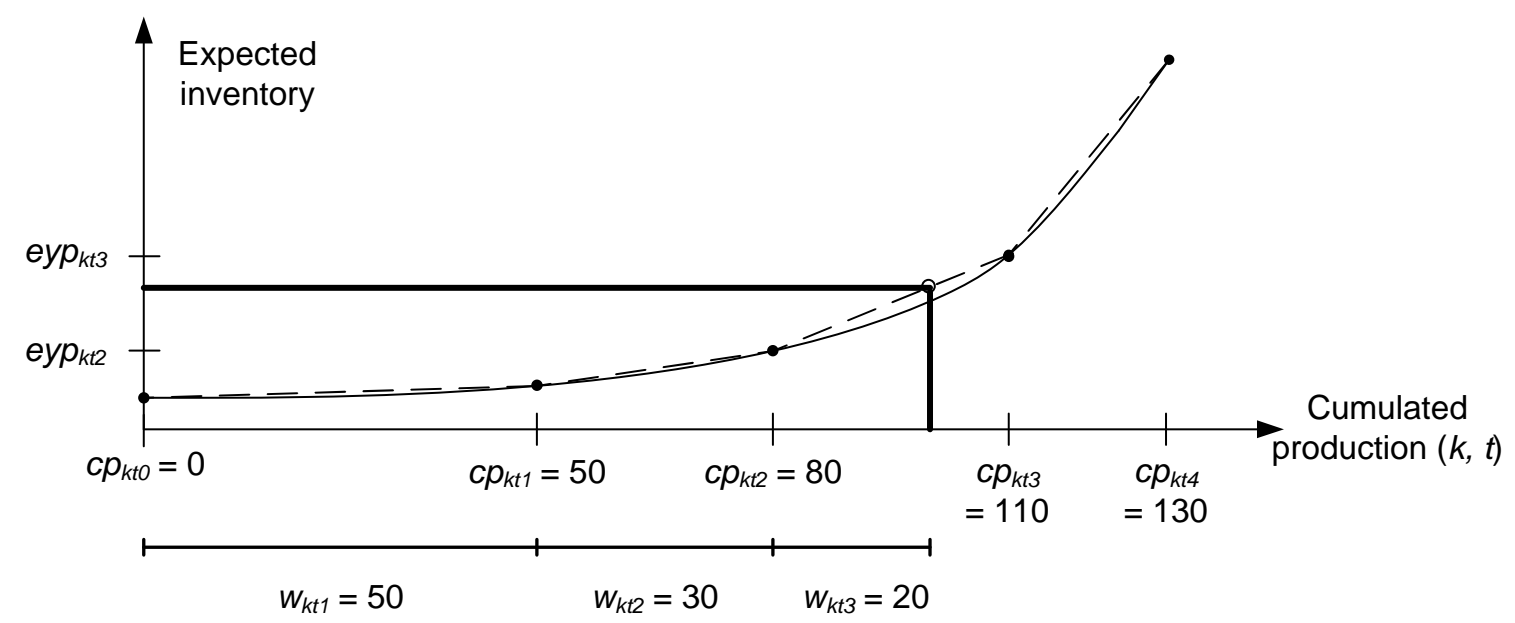

Figure 2: Linearization of the expected physical inventory function

an upper limit on the cumulated production $c p_{k t l}$ as shown in Figure 2. Assume that $l^{*}$ is the index of the segment that corresponds to the cumulated production of product $k$ in period $t$, i.e.,

$$
c p_{k t, l^{*}-1} \leq \sum_{\tau=1}^{t} q_{k \tau} \leq c p_{k t, l^{*}}
$$

In the example in Figure 2, we find $l^{*}=3$ and $c p_{k t, l^{*}-1}=80 \leq \sum_{\tau=1}^{t} q_{k \tau}=100 \leq$ $c p_{k t, l^{*}}=110$. Now we define $w_{k t l}$ as the part of the cumulated production quantity related to segment $l=1, \ldots, L$ of the linearization. Then the following two equations must hold:

$$
\begin{aligned}
\sum_{\tau=1}^{t} q_{k \tau} & =\sum_{l=1}^{l^{*}} w_{k t l} \\
\sum_{l=l^{*}+1}^{L} w_{k t l} & =0
\end{aligned}
$$

The segments 1 to $l^{*}-1$ are filled to their (maximum) "capacity" $w_{k t l}$ and pieces $l^{*}+1$ to $L$ must be zero:

$$
\begin{array}{rlrl}
w_{k t l} & =c p_{k t l}-c p_{k t, l-1}, & l & =1, \ldots, l^{*}-1 \\
w_{k t l} & =\sum_{\tau=1}^{t} q_{k \tau}-c p_{k t, l-1}, & l & =l^{*} \\
w_{k t l} & =0, & l & l=l^{*}+1, \ldots, L
\end{array}
$$

In the example in Figure 2, we hence have $w_{k t 1}=50, w_{k t 2}=30, w_{k t 3}=20$, and $w_{k t 4}=0$.

Note that the part of the cumulated production quantity $w_{k t l}$ that is assigned to segment $l$ of the linearization must not exceed the difference between the limits of cumulated production related to segment $l$, i.e.,

$$
w_{k t l} \leq c p_{k t l}-c p_{k t, l-1}, \quad \forall k, t, l
$$


In order to determine the supporting points for the approximation of $\mathrm{E}\left[B L_{k t}\right]$ and $\mathrm{E}\left[Y P_{k t}\right]$, it is necessary to determine for each value of cumulated production $c p_{k t l}$ the corresponding value of the expected backlog

$$
e b l_{k t l}=\mathrm{E}\left[\max \left(0 ; \sum_{\tau=1}^{t} D_{k \tau}-c p_{k t l}\right)\right]
$$

and the corresponding physical inventory:

$$
e y p_{k t l}=\mathrm{E}\left[\max \left(0 ; c p_{k t l}-\sum_{\tau=1}^{t} D_{k \tau}\right)\right]
$$

We now consider the special case that the period demand $D_{k t}$ is normally distributed with mean $\mathrm{E}\left[D_{k t}\right]$ and variance $\operatorname{VAR}\left[D_{k t}\right]$ estimated by a forecasting system. We furthermore assume that all demands are mutually independent, such that there is neither cross- nor autocorrelation. The cumulated demand $Z_{k t}=\sum_{\tau=1}^{t} D_{k \tau}$ has the mean $\mu_{Z_{k t}}=\sum_{\tau=1}^{t} \mathrm{E}\left[D_{k \tau}\right]$. As the demand is period-wise independent, we furthermore determine the standard deviation $\sigma_{Z_{k t}}=\sqrt{\sum_{\tau=1}^{t} \operatorname{VAR}\left[D_{k \tau}\right]}$. Let $\phi(x)$ and $\Phi(x)$ denote the density function and the probability function of a random variable $X$ following a standard normal distribution. Then the expected backlog and also the physical inventory corresponding to a specific (cumulated) production $c p_{k t l}$ can be computed via the "first-order loss function", see Tempelmeier (2006, p. 292).

$$
\begin{gathered}
e b l_{k t l}=\mathrm{E}\left[B L_{k t l}\right]=\sigma_{Z_{k t}} \cdot\left(\phi\left(\frac{c p_{k t l}-\mu_{Z_{k t}}}{\sigma_{Z_{k t}}}\right)-\frac{c p_{k t l}-\mu_{Z_{k t}}}{\sigma_{Z_{k t}}}\left[1-\Phi\left(\frac{c p_{k t l}-\mu_{Z_{k t}}}{\sigma_{Z_{k t}}}\right)\right]\right) \\
e y p_{k t l}=\mathrm{E}\left[Y P_{k t l}\right]=\sigma_{Z_{k t}} \cdot\left(\phi\left(\frac{c p_{k t l}-\mu_{Z_{k t}}}{\sigma_{Z_{k t}}}\right)+\frac{c p_{k t l}-\mu_{Z_{k t}}}{\sigma_{Z_{k t}}}\left[1-\Phi\left(-\frac{c p_{k t l}-\mu_{Z_{k t}}}{\sigma_{Z_{k t}}}\right)\right]\right)
\end{gathered}
$$

Let $e b l_{k t 0}$ and $e y p_{k t 0}$ denote the respective values corresponding to zero production. Hence $L+1$ different pairs $\left(c p_{k t l}, e b l_{k t l}\right)$ and $\left(c p_{k t l}, e y p_{k t l}\right)$ define the supporting points used to approximate the non-linear functions of expected backlog and inventory over (cumulated) production. Then the non-linear functions can be approximated as follows:

$$
\begin{aligned}
& \mathrm{E}\left[B L_{k t}\right] \approx e b l_{k t 0}+\sum_{l=1}^{L} \frac{e b l_{k t l}-e b l_{k t, l-1}}{c p_{k t l}-c p_{k t, l-1}} \cdot w_{k t l} \\
& \mathrm{E}\left[Y P_{k t}\right] \approx e y p_{k t 0}+\sum_{l=1}^{L} \frac{e y p_{k t l}-e y p_{k t, l-1}}{c p_{k t l}-c p_{k t, l-1}} \cdot w_{k t l}
\end{aligned}
$$

However, we have to ensure that the cumulated production in periods 1 to $t$ is assigned to the correct pieces of the linearization as required in Equations (33) to (35) and shown in the example in Figure 2.

If the period demand $D_{k t}$ follows a distribution type other than the normal distribution, the values of $e b l_{k t l}$ and $e y p_{k t l}$ in Equations (37) and (38) have to be determined based on the specific features of the respective demand distribution. The general principle of the piecewise linearization, however, still applies. 
Indices and index sets:

$\mathcal{L} \quad$ set of linearization segments $(l \in\{1, \ldots, L\})$

Deterministic parameters:

ebl $\quad$ expected backlog of product $k$ in period $t$ for segment $l$

$e b l_{k t 0} \quad$ expected backlog of product $k$ in period $t$ for zero cumulated production

$c p_{k t l} \quad$ cumulated production of product $k$ in period $t$ for segment $l$

eyptl $\quad$ expected physical inventory of product $k$ in period $t$ for segment $l$

$\operatorname{eyp}_{k t 0} \quad$ expected physical inventory of product $k$ in period $t$ for zero cumulated production

Decision variables:

$w_{k t l} \quad$ cumulated production quantity of product $k$ in periods 1 to $t$ assigned to stage $l$ of the linearization

\subsection{Formulation of the SCLSP-PLA}

With the additional notation in Table 3, the piecewise linear approximation SCLSP-PLA of the SCLSP can be defined as follows:

\section{SCLSP-PLA Model}

$$
\begin{aligned}
\min Z & =\sum_{k \in \mathcal{K}} \sum_{t \in \mathcal{T}} h c_{k} \cdot\left(\operatorname{eyp}_{k t 0}+\sum_{l=1}^{L} \frac{e y p_{k t l}-e y p_{k t, l-1}}{c p_{k t l}-c p_{k t, l-1}} \cdot w_{k t l}\right) \\
& +\sum_{k \in \mathcal{K}} \sum_{t \in \mathcal{T}} s c_{k} \cdot x_{k t}+\sum_{t \in \mathcal{T}} o c \cdot o_{t}
\end{aligned}
$$

subject to constraints (10), (11), (15), (17), (18), (19) and:

$$
\begin{array}{ll}
q_{k t}=\sum_{l=1}^{L} w_{k t l}-\sum_{l=1}^{L} w_{k, t-1, l}, & \forall k, t \\
w_{k t l} \leq c p_{k t l}-c p_{k t, l-1}, & \forall k, t, l \\
\sum_{t \in \mathcal{T}}\left(e b l_{k t 0}+\sum_{l=1}^{L} \frac{e b l_{k t l}-e b l_{k t, l-1}}{c p_{k t l}-c p_{k t, l-1}} \cdot w_{k t l}\right) \leq & \\
\left(1-\delta_{k}\right) \sum_{t \in \mathcal{T}}(T-t+1) \mathrm{E}\left[D_{k t}\right], & \forall k \\
w_{k t l} \geq 0, & \forall k, t, l
\end{array}
$$

In the objective function (43) and the $\delta$-service-level constraint (46) the linearized versions of the expected values of physical inventory and backlog are used. The production quantity per period equals the difference of cumulated production of subsequent periods, see (44). Constraint (45) was already introduced as (36). Finally, the production quantity assigned to segment $l$ of the linearization must be non-negative, see (47). 
It should be noted that due to the direction of optimization and the structure of the service-level constraint, the conditions in Equations (33) to (35) are always met! I.e., it is not necessary to define additional binary variables to ensure that cumulated production quantities $w_{k t l}$ are properly determined.

\section{A Fix-and-Optimize algorithm to solve the two ap- proximation models}

As the deterministic CLSP is already $\mathcal{N} \mathcal{P}$-hard (see Florian et al. (1980)), it tends to be difficult to solve the proposed approximations of the SCLSP to optimality in reasonable time. Therefore, we use an adapted version of the flexible Fix-and-Optimize heuristic proposed by Helber and Sahling (2010) as it leads to high-quality solutions for the multilevel version of the deterministic CLSP, see also Sahling et al. (2009) and Sahling (2010).

In the Fix-and-Optimize heuristic, a sequence of subproblems is solved to (sub-)optimality in an iterative fashion. In such a subproblem, the optimal values for a small number of binary setup variables are determined while the remaining binary setup variables are fixed to a given setup pattern from the solution of a previously solved subproblem. Furthermore, all real-valued decision variables are optimized in each subproblem as well. Due to the reduced number of binary setup variables, the time effort to solve the subproblem is moderate.

To apply the Fix-and-Optimize heuristic on the proposed approximations, we initially define the set $\mathcal{K} \mathcal{T}=\{1, \ldots, K\} \times\{1, \ldots, T\}$ of all product-period combinations $(k, t)$. Subsequently, the subset $\mathcal{K} \mathcal{T}^{\text {fix }} \subseteq \mathcal{K} \mathcal{T}$ can be defined. This subset $\mathcal{K} \mathcal{T}^{\text {fix }}$ contains those product-period combinations whose respective binary setup variables are fixed to a given setup pattern $\bar{x}_{k t}$ from the solution of a previous subproblem. Given the subset $\mathcal{K}^{\text {fix }}$, a subproblem can be defined by adding the following constraints to the SCLSP-SCN and the SCLSP-PLA as well as the benchmark CLSP-BT

$$
x_{k t}=\bar{x}_{k t}, \quad \forall(k, t) \in \mathcal{K} \mathcal{T}^{\text {fix }} .
$$

These additional constraints (48) assign a fixed setup pattern $\bar{x}_{k t}$ to those binary setup variables whose product-period combination $(k, t)$ belongs to the set $\mathcal{K} \mathcal{T}^{\text {fix }}$. Hence, the optimization of the binary setup variables is limited to those product-period combinations in the set $\mathcal{K} \mathcal{T}^{\text {opt }}=\mathcal{K} \mathcal{T} \backslash \mathcal{K} \mathcal{T}^{\text {fix }}$. We typically have $\left|\mathcal{K} \mathcal{T}^{\text {opt }}\right|<<\left|\mathcal{K} \mathcal{T}^{\text {fix }}\right|$. As an initial solution, we start similarly to Helber and Sahling (2010) with a lot-for-lot setup pattern, i. e. all binary setup variables $x_{k t}$ are fixed to 1 .

We use a product-oriented decomposition so that each subproblem corresponds to a single product. All binary setup variables of the respective product are optimized within the subproblem, while the others are fixed. Starting with the first product all products are considered once per iteration in the increasing sequence of the product index. The Fix-and-Optimize heuristic stops when a local optimum is reached, i.e., the last iteration yields no further improvement. 


\section{Numerical results}

\subsection{Accuracy of the different approximations}

\subsubsection{Description of the test instances}

For our numerical investigation, we defined 1,296 (artificial) test instances by systematically varying different parameters, e.g., the number of products and the number of periods. Table 4 gives an overview of the parameter values; details are presented in Appendix A.

Table 4: Parameters of the test instances

\begin{tabular}{lll}
\hline Number of products & $K$ & $=\{5,10,20\}$ \\
Number of periods & $T$ & $=\{5,10,20\}$ \\
Inter-period coefficient of variation of expected demand & $V C^{i p}$ & $=\{0.2,0.3\}$ \\
Demand coefficient of variation & $V C^{d}$ & $=\{0.1,0.3\}$ \\
Time between orders & $T B O$ & $=\{1,2,4\}$ \\
Utilization of resource due to processing & $U t i l$ & $=\{0.6,0.75\}$ \\
Setup time as fraction of period processing time & $t s^{\text {rel }}=\{0.0,0.25\}$ \\
Service-level goal & $\delta$ & $=\{0.8,0.9,0.95\}$ \\
Number of scenarios (SCLSP-SCN only) & $S$ & $=\{10,30,50\}$ \\
\hline
\end{tabular}

In order to create demand data that is both dynamic and stochastic, we first specified average demands $\mathrm{E}\left[D_{k}\right]$ for 20 different products $k$ as reported in Table 11 of the Appendix. To create dynamic time series of expected demand $\mathrm{E}\left[D_{k t}\right]$ per period $t$ around the mean $\mathrm{E}\left[D_{k}\right]$, we then drew (pseudo) random numbers from normal distributions with mean $\mathrm{E}\left[D_{k}\right]$ and standard deviation $V C^{i p} \cdot \mathrm{E}\left[D_{k}\right]$. In the case of the inter-period coefficient of demand $V C^{i p}=0.2$ this led to the dynamic time series with moderate time variability reported in Table 12. The more volatile time series for $V C^{i p}=0.3$ is given in Table 13 .

It is important to distinguish this inter-period variability of the expected demand $\mathrm{E}\left[D_{k t}\right]$ over the different periods $t=1, \ldots, T$ from the randomness of the demand $D_{k t}$ for a given $(k, t)$ combination. In our models in sections 3 to 5.2 , we require that the probability distribution of $D_{k t}$ is given for each $(k, t)$ combination. For our numerical study, we now assume that all random demands $D_{k t}$ follow a normal distribution with mean $\mathrm{E}\left[D_{k t}\right]$. We furthermore assume that the standard deviation of demand is time-invariant, proportional to the average expected demand

$$
\overline{\mathrm{E}\left[D_{k}\right]}=\frac{\sum_{t=1}^{T} \mathrm{E}\left[D_{k t}\right]}{T}
$$

and proportional to the demand coefficient of variation $V C^{d}$, i.e.,

$$
\sigma_{k t}=\sigma_{k}=\overline{\mathrm{E}\left[D_{k}\right]} \cdot V C^{d}
$$

We used this approach to model the usage of a properly designed demand forecasting system yielding point forecasts of the expected demand $\mathrm{E}\left[D_{k t}\right]$ and (as the most simple case) normally distributed forecasting errors with zero mean and time-invariant standard deviation, i.e., homoscedasticity of the forecasting residuals. Figure 3 shows examples of dynamic stochastic demand time series with time-invariant variance. The case with $V C^{d}=0.1\left(V C^{d}=0.3\right)$ models the use of a more (less) precise forecasting system, respectively. 


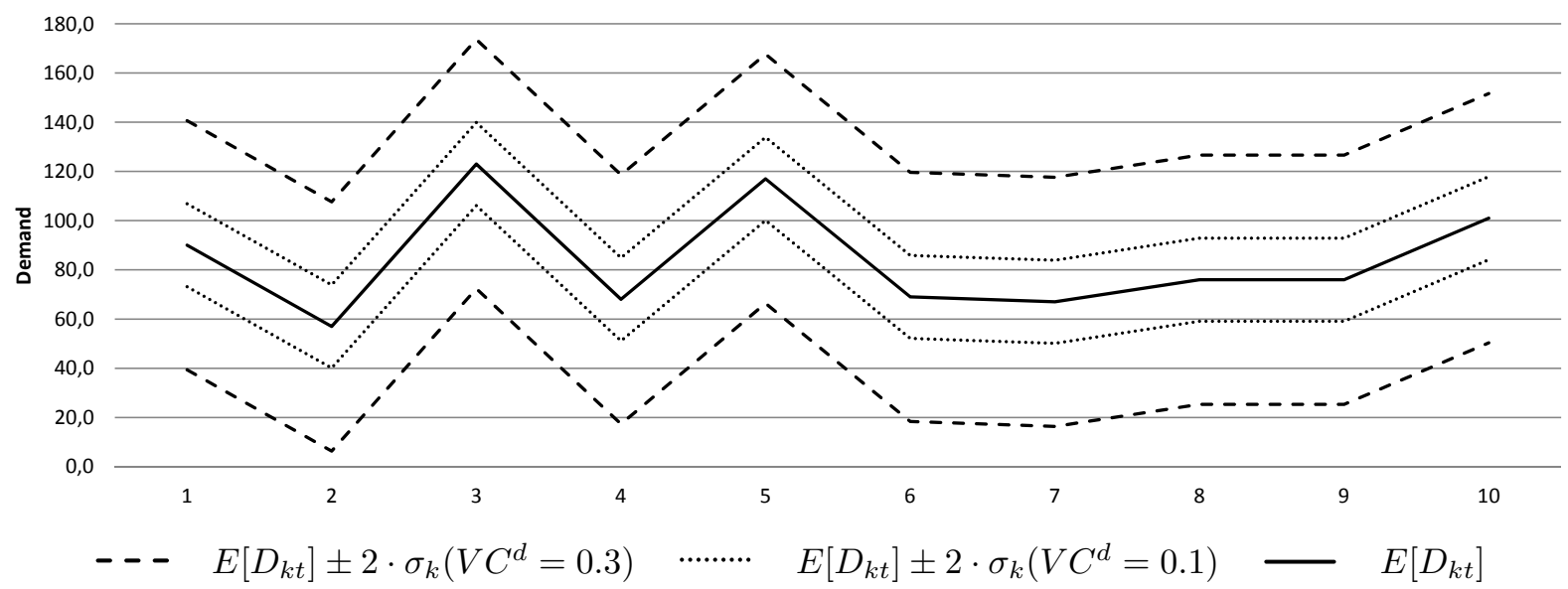

Figure 3: Demand of a product with lower and upper 95\% intervals for smaller $\left(V C^{d}=\right.$ $0.1)$ and higher $\left(V C^{d}=0.3\right)$ demand variability

To determine the scenario-specific demand $d_{k t}^{s}$ of product $k$ in period $t$ and scenario $s$ in the SCLSP-SCN in section 4, realizations were drawn from a normal distribution with expected value $\mathrm{E}\left[D_{k t}\right]$ and standard deviation $\sigma_{k}$ as specified in Equation (50) using either SRS or DS, see section 4.

\subsubsection{Analysis of the numerical results}

In our numerical experiments, we used an Intel Xeon CPU with $2.93 \mathrm{GHz}$ and $8 \mathrm{~GB}$ of RAM. The Fix-and-Optimize heuristic was implemented in the 64-bit version of GAMS 23.6. Each subproblem was solved by CPLEX 12.2 using two parallel threads. Because of the potentially large number of real-valued decision variables in both approximation models (number of scenarios in the SCLSP-SCN and linearization segments in the SCLSP-PLA), each subproblem optimization was aborted as soon as an integrality gap of $0.5 \%$ of the solution was reached or a time limit of 30 seconds was exceeded. If CPLEX did not find a mathematically feasible solution to the subproblem within this time limit, the time limit was extended to 300 seconds to find a solution. In the case of the SCLSP-PLA, we operated with 10 linearization segments which are specific for each product-period combination $(k, t)$ and distributed as shown in Figure 1 to relate expected physical inventory and backlog to cumulated production.

In Table 5, we illustrate the average results over all 1,296 test instances for the scenario approach SCLSP-SCN based on Simple Random Sampling (SRS) and Descriptive Sampling (DS), the piecewise linear approximation model SCLSP-PLA and, as a benchmark, the modified Bookbinder and Tan approach CLSP-BT as described at the end of section 3. In the first row we address the service level (SL) and report the percentage of problem instances for which the required $\delta$-service level is met for all products in this instance. Remember that for given production quantities, the expected backlog and hence also the $\delta$-service level can easily be determined, either exactly (e.g., in our case for normally distributed demand) or by approximating any theoretical demand distribution by an empirical distribution. In the next two rows, we report the fraction of problem instances for which all products in this instance missed the required service level by at most 1 or 2 percentage points, respectively. E.g., if the SCLSP-SCN based on Simple Random Sampling (SRS) was solved using 10 scenarios, $61.5 \%$ of the problem instances had solutions which missed the required $\delta$-service level for all products by at most 2 per- 
centage points, whereas this holds true for $100 \%$ of the problem instances if Descriptive Sampling is used. The results indicate that DS leads to a substantial variance reduction, here with respect to the aggregate service level.

Note that the SCLSP-PLA as well as the CLSP-BT always met the service-level target for all products, while the SCLSP-SCN almost always failed to meet the target exactly due to the variance of the estimated $\delta$-service level. In the next two rows we compare only those solutions to test instances for which all products met the required $\delta$-service-level target and first report the percentage of best solutions "BSol" determined by the respective model. The percentage of best solutions found by the SCLSP-PLA is exorbitantly high with $90.43 \%$ compared to values between $0.08 \%$ and $2.78 \%$ for the SCLSP-SCN and 4.86 $\%$ for the CLSP-BT. This is due to the fact that we only consider those solutions which meet the required $\delta$-service level for all products in the instance.

The average deviation "DevBSol" from the best known solution is almost always very small, except for the benchmark CLSP-BT which delivers a very high deviation. The reason for this very high deviation is that a constraint on the $\delta$-service level for each period is imposed in the CLSP-BT, see equation (5). The SCLSP, however, operates with an aggregate service level constraint only. The period-specific constraint can lead to a substantial increase of the number of setups and hence the setup costs. The final three rows "SLP" to "SLP-2\%" report over all instances the average of the percentage of the number of products for which the $\delta$-service level was met or missed by at most $1 \%$ or $2 \%$, respectively. On average, $80.44 \%$ of the products in the solutions of the SCLSP-SCN: SRS for 10 scenarios either met the required $\delta$-service level or missed it by at most 1 percentage point. The comparison of between SRS and DS shows again the strong variance reduction due to DS. With respect to the fraction of products that missed the target service level by at most 1 percentage point, DS with only 10 scenarios led to a fraction of $98.46 \%$ and hence even outperformed SRS with 50 scenarios and a fraction of only $95.07 \%$. The overall numerical results indicate that the SCLSP-PLA is clearly superior to the SCLSP-SCN, but the latter still delivers solutions that can be considered to be robust from a practical point of view.

Table 5: Overall numerical results for all test instances

\begin{tabular}{|l|c|c|c|c|c|c|c|c|}
\hline & \multicolumn{3}{|c|}{ SCLSP-SCN: SRS } & \multicolumn{3}{c|}{ SCLSP-SCN: DS } & SCLSP- & CLSP- \\
& $|S|=10$ & $|S|=30$ & $|S|=50$ & $|S|=10$ & $|S|=30$ & $|S|=50$ & PLA & BT \\
\hline SL [\%] & 0.77 & 4.71 & 1.08 & 1.77 & 1.47 & 0.77 & 100.00 & 100.00 \\
SL-1\% [\%] & 35.26 & 64.74 & 72.53 & 87.65 & 100.00 & 99.85 & 100.00 & 100.00 \\
SL-2\% [\%] & 61.50 & 88.12 & 98.15 & 100.00 & 100.00 & 100.00 & 100.00 & 100.00 \\
\hline BSol [\%] & 0.08 & 2.78 & 0.46 & 0.85 & 0.39 & 0.15 & 90.43 & 4.86 \\
DevBSol [\%] & 2.51 & 0.58 & 1.78 & 1.23 & 1.09 & 0.76 & 1.24 & 105.72 \\
\hline SLP [\%] & 49.24 & 60.10 & 48.56 & 47.31 & 48.86 & 42.92 & 100.00 & 100.00 \\
SLP-1\% [\%] & 80.44 & 93.28 & 95.07 & 98.46 & 100.00 & 99.99 & 100.00 & 100.00 \\
SLP-2\% [\%] & 92.92 & 98.46 & 99.88 & 100.00 & 100.00 & 100.00 & 100.00 & 100.00 \\
\hline
\end{tabular}

As the DS generates much better results than the SRS, we only report further results for DS which explains why percentages in the following tables do not necessarily add up to $100 \%$. In Table 6, a detailed analysis with respect to the number of products and periods is given. An entry "**** indicates that a quantity could not be computed as not a single instance met the service level constraint for all products. For the SCLSP$\mathrm{SCN}$, the solution quality tends to be higher for smaller test instances. In the case of the SCLSP-SCN, the fraction of solutions which met the required $\delta$-service level for all products decreases with the number of products and periods. Only for at most $3.7 \%$ of 
Table 6: Numerical results for all test instances based on the number of products and periods

\begin{tabular}{|l|l|c|c|c|c|c|c|}
\hline & & $K=5$ & $K=10$ & $K=20$ & $T=5$ & $T=10$ & $T=20$ \\
\hline SL [\%] & DS, $|S|=10$ & 3.70 & 1.39 & 0.23 & 2.55 & 1.16 & 1.62 \\
& DS, $|S|=30$ & 2.55 & 1.62 & 0.23 & 2.55 & 0.69 & 1.16 \\
& DS, $|S|=50$ & 2.08 & 0.23 & 0.00 & 1.39 & 0.00 & 0.93 \\
& SCLSP-PLA & 100.00 & 100.00 & 100.00 & 100.00 & 100.00 & 100.00 \\
& CLSP-BT & 100.00 & 100.00 & 100.00 & 100.00 & 100.00 & 100.00 \\
\hline SL-1\% [\%] & DS, $|S|=10$ & 92.82 & 92.59 & 77.55 & 74.54 & 90.74 & 97.69 \\
& DS, $|S|=30$ & 100.00 & 100.00 & 100.00 & 100.00 & 100.00 & 100.00 \\
& DS, $|S|=50$ & 100.00 & 100.00 & 99.54 & 99.54 & 100.00 & 100.00 \\
& SCLSP-PLA & 100.00 & 100.00 & 100.00 & 100.00 & 100.00 & 100.00 \\
& CLSP-BT & 100.00 & 100.00 & 100.00 & 100.00 & 100.00 & 100.00 \\
\hline BSol [\%] & DS, $|S|=10$ & 1.62 & 0.69 & 0.23 & 1.62 & 0.69 & 0.23 \\
& DS, $|S|=30$ & 0.69 & 0.23 & 0.23 & 0.93 & 0.23 & 0.00 \\
& DS, $|S|=50$ & 0.46 & 0.00 & $* * *$ & 0.00 & $* * *$ & 0.46 \\
& SCLSP-PLA & 85.19 & 91.44 & 94.68 & 83.80 & 94.44 & 93.06 \\
& CLSP-BT & 4.86 & 5.09 & 4.63 & 6.25 & 4.17 & 4.17 \\
\hline DevBSol [\%] & DS, $|S|=10$ & 1.32 & 1.18 & 0.00 & 0.50 & 1.21 & 2.38 \\
& DS, $|S|=30$ & 1.13 & 1.18 & 0.00 & 1.03 & 0.58 & 1.55 \\
& DS, $|S|=50$ & 0.70 & 1.25 & $* * *$ & 0.66 & $* * *$ & 0.90 \\
& SCLSP-PLA & 1.30 & 1.21 & 1.20 & 1.95 & 1.28 & 0.47 \\
& CLSP-BT & 103.20 & 104.73 & 109.23 & 52.75 & 92.56 & 171.86 \\
\hline SLP-1\% [\%] & DS, $|S|=10$ & 53.80 & 46.99 & 41.13 & 48.45 & 47.27 & 46.20 \\
& DS, $|S|=10$ & 98.56 & 98.84 & 97.97 & 96.23 & 99.27 & 99.88 \\
& DS, $|S|=30$ & 100.00 & 100.00 & 100.00 & 100.00 & 100.00 & 100.00 \\
& DS, $|S|=50$ & 100.00 & 100.00 & 99.98 & 99.98 & 100.00 & 100.00 \\
& SCLSP-PLA & 100.00 & 100.00 & 100.00 & 100.00 & 100.00 & 100.00 \\
& DS, $|S|=30$ & 45.32 & 56.69 & 44.57 & 54.02 & 47.40 & 45.17 \\
& DS, $|S|=50$ & 43.15 & 43.63 & 41.99 & 47.07 & 38.37 & 43.33 \\
& SCLSP-PLA & 100.00 & 100.00 & 100.00 & 100.00 & 100.00 & 100.00 \\
& SLSP-BT & 100.00 & 100.00 & 100.00 & 100.00 & 100.00 & 100.00 \\
\hline SLP [\%] & 100.00 & 100.00 & 100.00 & 100.00 & 100.00 & 100.00 \\
\hline
\end{tabular}


the test instances with $K=5$, a solution that is feasible for all products has been found. This portion decreases to $0.23 \%$ for test instances with 20 products. The influence of the number of periods is less distinct. From the perspective of individual products, it is interesting that for at least $40 \%$ of the products the required service-level has been reached. If it has not been reached, it has usually been missed by at most one percentage point. Again the huge average deviation "DevBSol" of the CLSP-BT is eye-catching, in particular for larger number of periods.

In Table 7, numerical results are presented with respect to the variability of demand (reflecting the forecast error) and the target $\delta$-service level. While the SCLSP-PLA again performs extremely well under any conditions, the solution quality of the SCLSP-SCN seems to deteriorate as the demand variability or the target service-level increase. During our numerical investigation, we realized that the influence of the remaining parameters on the quality of the solutions is limited.

Table 8 reports the average run time of the Fix-and-Optimize algorithm for the different model variants. The solution times tend to increase with the number of products, periods, and-in case of the SCLSP-SCN-with the number of scenarios. Furthermore, the piecewise linear approximation model SCLSP-PLA appears to outperform the SCLSPSCN from the computational perspective as well. For example, a test instance with 5 products and 20 periods can be solved using the SCLSP-PLA in 16 seconds on average. By contrast, the solution of the SCLSP-SCN with 50 scenarios and DS requires on average 185 seconds. The very fast solutions times of the CLSP-BT are attended by the costly results discussed earlier.

Based on this analysis, the reader might be tempted to conclude that the SCLSP-SCN is essentially useless. However, it should be noted that the scenario approach offers some kind of flexibility that does not exist in the other approximation model SCLSP-PLA: It is possible to model within a scenario $s$ of the SCLSP-SCN a probabilistic dependency of the demand for two different product-period combinations $(k, t)$ and $(\hat{k}, \hat{t})$, respectively. In our problem specification in section 2.1, we explicitly ruled this possibility out, but it might be relevant in a practical setting and we do not see a way to incorporate it in the SCLSP-PLA.

\subsection{Impact of forecast (in-)accuracy on costs and planned $\mathrm{dy}$ - namic safety stocks}

From the planner's perspective, the randomness of demand is reflected in the errors of the forecasts from the forecasting system. A large standard deviation $\sigma_{k}$ of the demand of product $k$ leads to a large standard error of the demand forecast. For this reason, we now study the impact of demand variability (as the source of forecasting inaccuracy) on costs and safety stocks.

To this end, we consider from Table 4 the six cases with $K=5, T=10, V C^{i p}=0.3$, $U t i l=0.75, t s^{r e l}=0.25$, and now a target $\delta$-service level of either $95 \%$ or $99 \%$. Each test instance was solved to optimality using the SCLSP-PLA. The total costs of each solution are given for each combination of $T B O$ and $V C^{d}$ in Tables 9 and 10. In both tables, we report the relative cost increase due to the increased demand variability (or forecast inaccuracy).

As the setup costs (and hence the TBO) increase, the total costs also increase. They furthermore rise with the target $\delta$-service level or the demand variability. The relative growth of the costs due to a higher demand variability is much stronger for low setup costs $(T B O=1)$ than for high setup costs $(T B O=4)$. The phenomenon is particularly 
Table 7: Solution quality for all test instances based on $V C^{d}$ and the given $\delta$-service level

\begin{tabular}{|l|l|c|c|c|c|c|}
\hline & & $V C^{d}=0.1$ & $V C^{d}=0.3$ & $\delta=0.80$ & $\delta=0.90$ & $\delta=0.95$ \\
\hline SL [\%] & DS, $|S|=10$ & 2.62 & 0.93 & 4.86 & 0.46 & 0.00 \\
& DS, $|S|=30$ & 2.16 & 0.77 & 4.17 & 0.23 & 0.00 \\
& DS, $|S|=50$ & 1.23 & 0.31 & 1.62 & 0.69 & 0.00 \\
& SCLSP-PLA & 100.00 & 100.00 & 100.00 & 100.00 & 100.00 \\
& CLSP-BT & 100.00 & 100.00 & 100.00 & 100.00 & 100.00 \\
SL-1\% [\%] & DS, $|S|=10$ & 100.00 & 75.31 & 94.91 & 85.42 & 82.64 \\
& DS, $|S|=30$ & 100.00 & 100.00 & 100.00 & 100.00 & 100.00 \\
& DS, $|S|=50$ & 100.00 & 99.69 & 100.00 & 100.00 & 99.54 \\
& SCLSP-PLA & 100.00 & 100.00 & 100.00 & 100.00 & 100.00 \\
& CLSP-BT & 100.00 & 100.00 & 100.00 & 100.00 & 100.00 \\
\hline BSol [\%] & DS, $|S|=10$ & 1.08 & 0.62 & 2.55 & 0.00 & $* * *$ \\
& DS, $|S|=30$ & 0.62 & 0.15 & 0.93 & 0.23 & $* * *$ \\
& DS, $|S|=50$ & 0.15 & 0.15 & 0.46 & 0.00 & $* * *$ \\
& SCLSP-PLA & 93.98 & 86.88 & 88.43 & 97.45 & 85.42 \\
& CLSP-BT & 0.00 & 9.72 & 0.00 & 0.00 & 14.58 \\
DevBSol [\%] & DS, $|S|=10$ & 0.88 & 2.22 & 1.28 & 0.68 & $* * *$ \\
& DS, $|S|=30$ & 0.84 & 1.81 & 1.15 & 0.00 & $* * *$ \\
& DS, $|S|=50$ & 0.83 & 0.49 & 0.85 & 0.55 & $* * *$ \\
& SCLSP-PLA & 0.07 & 2.40 & 0.21 & 0.02 & 3.48 \\
& CLSP-BT & 121.56 & 89.88 & 190.96 & 81.74 & 44.47 \\
\hline SLP [\%] & DS, $|S|=10$ & 51.47 & 43.15 & 57.34 & 44.16 & 40.43 \\
& DS, $|S|=30$ & 49.72 & 48.00 & 55.69 & 46.10 & 44.79 \\
& DS, $|S|=50$ & 44.39 & 41.46 & 50.34 & 40.93 & 37.51 \\
& SCLSP-PLA & 100.00 & 100.00 & 100.00 & 100.00 & 100.00 \\
& CLSP-BT & 100.00 & 100.00 & 100.00 & 100.00 & 100.00 \\
& DS, $|S|=10$ & 100.00 & 96.92 & 99.54 & 98.10 & 97.74 \\
SLP-1\% [\%] & DS, $|S|=30$ & 100.00 & 100.00 & 100.00 & 100.00 & 100.00 \\
& DS, $|S|=50$ & 100.00 & 99.98 & 100.00 & 100.00 & 99.98 \\
& SCLSP-PLA & 100.00 & 100.00 & 100.00 & 100.00 & 100.00 \\
& CLSP-BT & 100.00 & 100.00 & 100.00 & 100.00 & 100.00 \\
\hline
\end{tabular}


Table 8: Average solution time (in CPU seconds) for all test instances

\begin{tabular}{|l|l|c|c|c|}
\hline & & $T=5$ & $T=10$ & $T=20$ \\
\hline$K=5$ & DS, $|S|=10$ SCN: SRS & 1,08 & 4,32 & 19,83 \\
& DS, $|S|=30$ SCN: SRS & 3,64 & 21,00 & 105,66 \\
& DS, $|S|=50$ SCN: SRS & 7,58 & 46,00 & 262,73 \\
& DS, $|S|=10$ SCN: DS & 1,07 & 4,47 & 19,41 \\
& DS, $|S|=30$ SCN: DS & 3,64 & 20,00 & 103,44 \\
& DS, $|S|=50$ SCN: DS & 7,34 & 44,01 & 246,88 \\
& SCLSP-PLA & 0,51 & 1,90 & 16,05 \\
& CLSP-BT & 0,20 & 0,34 & 0,61 \\
\hline$K=10$ & DS, $|S|=10$ SCN: SRS & 13,82 & 81,18 & 575,25 \\
& DS, $|S|=30$ SCN: SRS & 3,77 & 17,71 & 112,13 \\
& DS, $|S|=50$ SCN: SRS & 30,10 & 186,80 & 1381,87 \\
& DS, $|S|=10$ SCN: DS & 3,74 & 18,17 & 102,06 \\
& DS, $|S|=30$ SCN: DS & 14,70 & 83,80 & 577,03 \\
& DS, $|S|=50$ SCN: DS & 30,92 & 190,51 & 1401,26 \\
& SCLSP-PLA & 1,64 & 6,61 & 85,95 \\
& CLSP-BT & 0,57 & 0,87 & 2,41 \\
\hline$K=20$ & DS, $|S|=10$ SCN: SRS & 15,67 & 70,80 & 390,30 \\
& DS, $|S|=30$ SCN: SRS & 71,93 & 367,86 & 2156,08 \\
& DS, $|S|=50$ SCN: SRS & 174,84 & 859,91 & 5338,12 \\
& DS, $|S|=10$ SCN: DS & 16,72 & 69,55 & 372,68 \\
& DS, $|S|=30$ SCN: DS & 76,07 & 388,38 & 2265,90 \\
& DS, $|S|=50$ SCN: DS & 185,06 & 850,47 & 4910,22 \\
& SCLSP-PLA & 5,04 & 24,60 & 310,96 \\
& CLSP-BT & 1,20 & 3,29 & 5,90 \\
\hline \multirow{1}{*}{$K=$} & & & \\
\hline
\end{tabular}

Table 9: Total costs of test instances $(\delta=0.95)$

\begin{tabular}{|l|c|c|c|}
\hline & $V C^{d}=0.1$ & $V C^{d}=0.3$ & Rel. Increase \\
\hline$T B O=1$ & 1806.47 & 2969.09 & $64.36 \%$ \\
$T B O=2$ & 5066.85 & 6007.12 & $18.56 \%$ \\
$T B O=4$ & 13008.63 & 14265.49 & $9.66 \%$ \\
\hline
\end{tabular}

Table 10: Total costs of test instances $(\delta=0.99)$

\begin{tabular}{|l|c|c|c|}
\hline & $V C^{d}=0.1$ & $V C^{d}=0.3$ & Rel. Increase \\
\hline$T B O=1$ & 2758.99 & 12343.53 & $347.39 \%$ \\
$T B O=2$ & 7027.14 & 17134.86 & $143.84 \%$ \\
$T B O=4$ & 19548.03 & 33527.46 & $71.51 \%$ \\
\hline
\end{tabular}

dramatic if a very high service level is demanded $(\delta=99 \%)$. This result shows that as setup costs and setup times are reduced in an attempt to achieve "lean production", it becomes c.p. more important to have an accurate forecasting system, i.e., to reduce the perceived demand variability.

We finally address the topic of dynamic safety stocks by studying Product 4 (out of the five products) in more detail. The expected demand per period as well as the lower and upper limits of the $95 \%$ demand intervals are depicted in Figure 3. 
In Figure 4 we present for a target $\delta$-service level of $99 \%$ the production quantities, the expected physical inventory, the expected backlog and the safety stocks as defined in Equation (20) for the case of a $T B O=4$. The figures show that the overall expected physical inventory and the safety stock is substantially higher for the case with high demand variability $\left(V C^{d}=0.3\right)$ than for the case with low demand variability $\left(V C^{d}=\right.$ 0.1). The safety stocks are clearly dynamic and differ from lot to lot. They can also be negative, as in Periods 1 and 2, so that they lead to a substantial planned backlog in Period 2.

\section{Conclusions, managerial insights, and outlook}

In this paper, we have proposed and evaluated different models to coordinate production quantities and safety stocks subject to random demand and a capacity-constrained production system. The solution of the models leads to robust production plans that contain dynamic safety stocks. We have furthermore presented a well-defined backlog-oriented $\delta$-service level measure that overcomes the deficiencies of the $\gamma$-service level measure. It offers a clear interpretation and can in addition be directly related to the average waiting time of the demands. It can also be easily incorporated into dynamic and stochastic production planning models like the SCLSP. A Fix-and-Optimize heuristic was used to solve the two numerically tractable approximation models for the SCLSP as well as a model variant with period-specific service level constraints based on ideas suggested by Bookbinder and Tan (1988). Our numerical study shows that the model variant based on a piecewise linear approximation of the non-linear functions of expected backlog and expected physical inventory performs particularly well. The other modeling approach based on a scenario technique turns out to be less accurate, but more flexible with respect to probabilistic dependencies of the demands within a single scenario. To determine demand realizations for the scenario approach, Descriptive Sampling as proposed by Saliby (1990) clearly outperformed a Simple Random Sampling approach and showed a surprisingly high degree of accuracy even for small numbers of scenarios.

One important managerial insight can be derived from the comparison of the numerical results of the CLSP-model inspired by Bookbinder and Tan to enforce a period-specific $\delta$-service level measure as opposed to an aggregate multi-period $\delta$-service level measure in our approximation models: Imposing a period-specific service constraint can be much more expensive than imposing only an aggregate service level constraint and tolerating occasional backlogs. If the management is mainly interested in robust schedules that minimize system nervousness and bullwhip effects while meeting aggregate service level constraints, the models presented in this paper turn out to be very useful to determine such schedules. Our numerical examples have furthermore shown the potentially strong impact of demand variability (or forecast inaccuracy) on safety stocks and the costs of the schedule. We have in addition emphasized the dynamic nature of safety stocks in the case of dynamic demand and production quantities as well as the value of precise demand forecasts for production systems operating with small production lots and short production cycles. Our models can finally also be used to quantify the benefit from an improved forecasting accuracy, which is in our eyes an important indirect managerial aspect of our work.

Future research should address the case of multi-level supply chains and/or additional

randomness due to random production capacities and the use of the models within a rolling horizon context. 


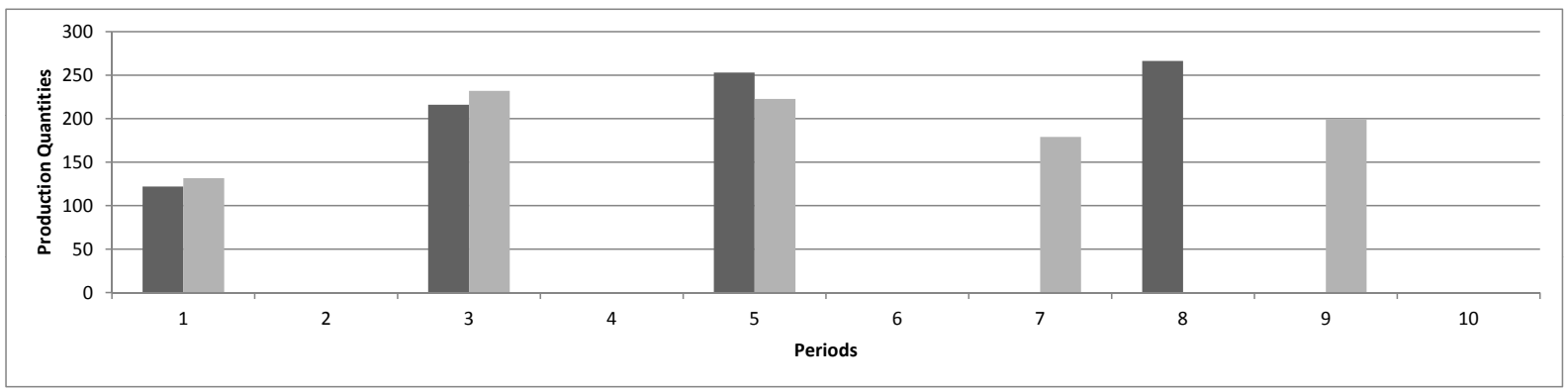

(a) Production Quantities

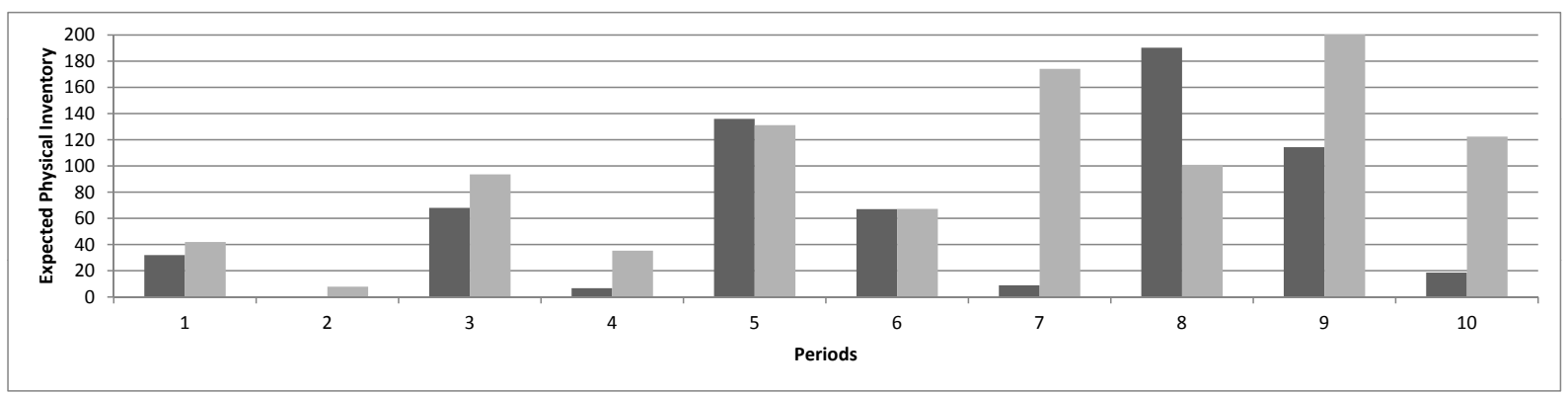

(b) Expected Physical Inventory

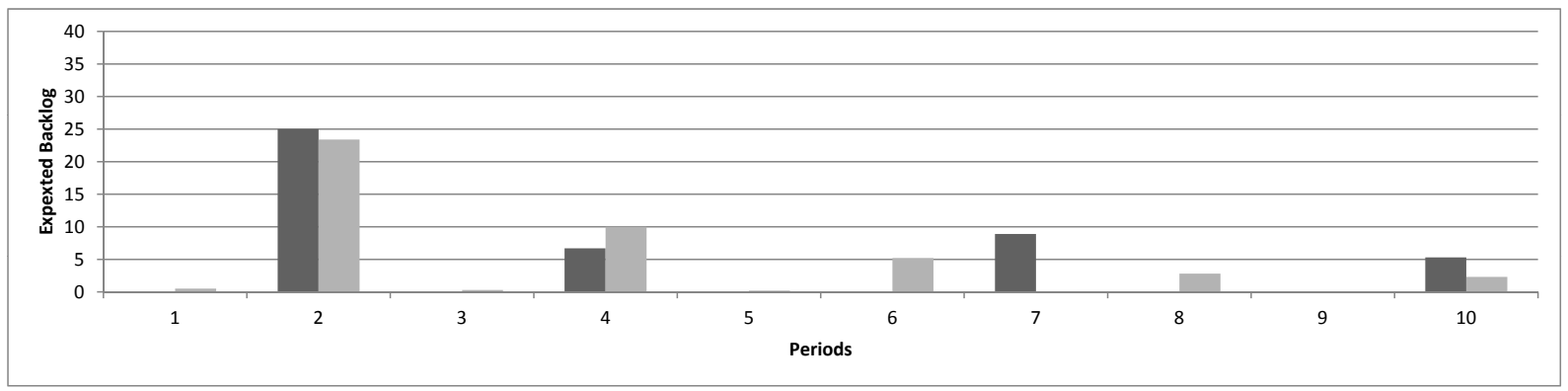

(c) Expected Backlog

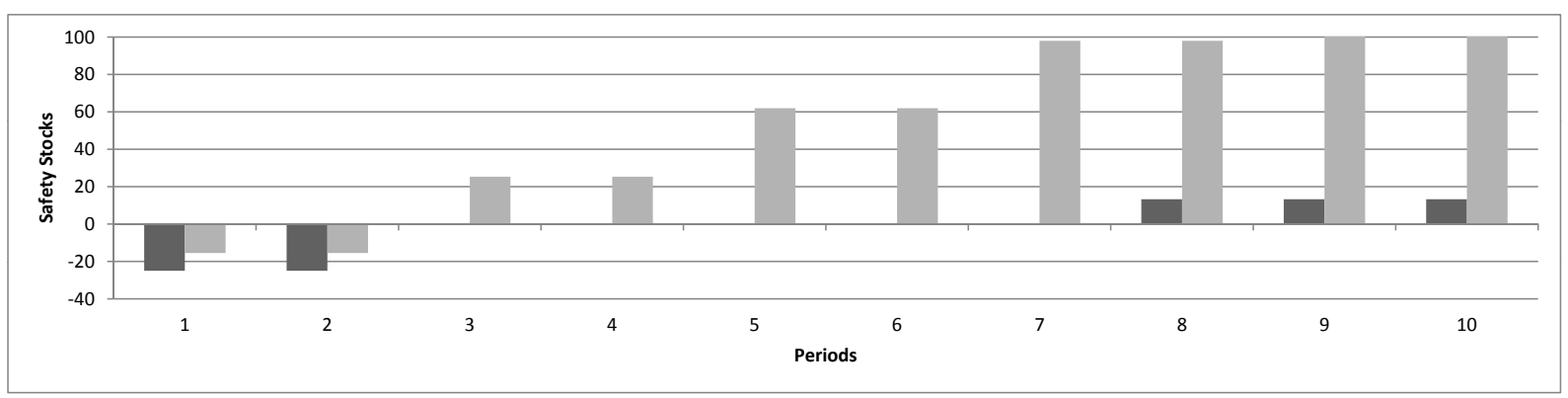

(d) Safety Stocks

$$
V C^{d}=0.1 \quad \square C^{d}=0.3
$$

Figure 4: $T B O=4$ and $\delta=0.99$

\section{A Test sets}

Table 11 reports the mean $\mathrm{E}\left[D_{k}\right]$ of the demand. For each product $k$ and period $t$, the expected dynamic demand $E\left[D_{k t}\right]$ is drawn from a normal distribution with mean $\mathrm{E}\left[D_{k}\right]$ and standard deviation $V C^{i p} \cdot \mathrm{E}\left[D_{k}\right]$. The drawn demand series are given in Table 12 for $V C^{i p}=0.2$ and in Table 13 for $V C^{i p}=0.3$.

For a problem instance from Table 4 with $K$ products and $T$ periods, the first $K$ rows 
Table 11: Series of expected demand $E\left[D_{k}\right]$

\begin{tabular}{|c|c|c|c|c|c|c|c|c|c|c|c|c|c|c|c|c|c|c|c|c|c|c|c|}
\hline$k$ & 1 & 2 & 3 & 4 & 5 & 6 & 7 & 8 & 9 & 10 & 11 & 12 & 13 & 14 & 15 & 16 & 17 & 18 & 19 & 20 \\
\hline $\mathrm{E}\left[D_{k}\right]$ & 67 & 135 & 105 & 80 & 79 & 72 & 85 & 136 & 56 & 100 & 150 & 108 & 150 & 126 & 63 & 114 & 66 & 75 & 117 & 93 \\
\hline
\end{tabular}

Table 12: Series of expected demand $E\left[D_{k t}\right]$ for $V C^{i p}=0.2$

\begin{tabular}{|c|c|c|c|c|c|c|c|c|c|c|c|c|c|c|c|c|c|c|c|c|}
\hline$k \backslash t$ & 1 & 2 & 3 & 4 & 5 & 6 & 7 & 8 & 9 & 10 & 11 & 12 & 13 & 14 & 15 & 16 & 17 & 18 & 19 & 20 \\
\hline 1 & 74 & 64 & 77 & 42 & 70 & 51 & 83 & 48 & 89 & 80 & 77 & 67 & 41 & 66 & 69 & 89 & 85 & 78 & 80 & 71 \\
\hline 2 & 140 & 99 & 128 & 120 & 111 & 83 & 109 & 140 & 99 & 77 & 94 & 141 & 164 & 95 & 133 & 131 & 152 & 120 & 114 & 90 \\
\hline 3 & 88 & 105 & 95 & 78 & 122 & 156 & 88 & 78 & 147 & 146 & 93 & 101 & 111 & 103 & 79 & 102 & 95 & 77 & 105 & 102 \\
\hline 4 & 67 & 82 & 95 & 113 & 82 & 77 & 75 & 60 & 91 & 85 & 65 & 71 & 83 & 73 & 68 & 111 & 77 & 47 & 97 & 77 \\
\hline 5 & 93 & 69 & 80 & 79 & 103 & 100 & 80 & 60 & 96 & 61 & 68 & 98 & 67 & 85 & 87 & 77 & 52 & 72 & 58 & 52 \\
\hline 6 & 79 & 70 & 87 & 52 & 93 & 96 & 73 & 77 & 81 & 93 & 86 & 66 & 76 & 83 & 61 & 50 & 74 & 71 & 70 & 81 \\
\hline 7 & 103 & 104 & 90 & 73 & 75 & 94 & 94 & 91 & 76 & 106 & 95 & 107 & 100 & 115 & 71 & 87 & 74 & 52 & 78 & 71 \\
\hline 8 & 144 & 151 & 126 & 135 & 158 & 175 & 97 & 100 & 104 & 119 & 130 & 196 & 115 & 134 & 202 & 97 & 141 & 100 & 71 & 143 \\
\hline 9 & 56 & 61 & 45 & 55 & 45 & 43 & 24 & 57 & 40 & 58 & 66 & 58 & 58 & 52 & 58 & 50 & 71 & 64 & 54 & 51 \\
\hline 10 & 107 & 127 & 118 & 97 & 106 & 123 & 97 & 85 & 94 & 85 & 91 & 87 & 67 & 115 & 137 & 109 & 91 & 108 & 137 & 60 \\
\hline 11 & 131 & 172 & 163 & 136 & 118 & 192 & 204 & 142 & 188 & 167 & 123 & 166 & 147 & 129 & 174 & 75 & 148 & 132 & 148 & 122 \\
\hline 12 & 84 & 90 & 122 & 124 & 65 & 118 & 94 & 145 & 85 & 130 & 102 & 71 & 109 & 102 & 89 & 71 & 109 & 73 & 84 & 127 \\
\hline 13 & 137 & 152 & 110 & 136 & 129 & 138 & 123 & 128 & 63 & 193 & 140 & 147 & 159 & 129 & 91 & 182 & 106 & 177 & 96 & 179 \\
\hline 14 & 128 & 139 & 125 & 130 & 84 & 105 & 131 & 82 & 139 & 106 & 136 & 85 & 131 & 121 & 116 & 98 & 163 & 102 & 100 & 138 \\
\hline 15 & 52 & 99 & 50 & 44 & 45 & 55 & 63 & 51 & 78 & 77 & 55 & 73 & 65 & 62 & 59 & 48 & 69 & 43 & 67 & 81 \\
\hline 16 & 85 & 135 & 93 & 130 & 111 & 89 & 112 & 177 & 103 & 159 & 77 & 121 & 113 & 105 & 110 & 112 & 137 & 129 & 102 & 94 \\
\hline 17 & 68 & 76 & 52 & 66 & 71 & 71 & 54 & 62 & 70 & 55 & 55 & 66 & 92 & 53 & 59 & 56 & 54 & 66 & 59 & 82 \\
\hline 18 & 85 & 66 & 109 & 96 & 59 & 66 & 76 & 68 & 65 & 90 & 80 & 84 & 90 & 83 & 54 & 73 & 70 & 86 & 74 & 91 \\
\hline 19 & 92 & 115 & 104 & 98 & 120 & 91 & 156 & 119 & 69 & 70 & 122 & 80 & 72 & 74 & 137 & 92 & 124 & 140 & 84 & 107 \\
\hline 20 & 93 & 94 & 114 & 98 & 66 & 94 & 107 & 83 & 109 & 99 & 94 & 93 & 81 & 77 & 90 & 85 & 92 & 95 & 91 & 128 \\
\hline
\end{tabular}

Table 13: Series of expected demand $E\left[D_{k t}\right]$ for $V C^{i p}=0.3$

\begin{tabular}{|c|c|c|c|c|c|c|c|c|c|c|c|c|c|c|c|c|c|c|c|c|}
\hline$k \backslash t$ & 1 & 2 & 3 & 4 & 5 & 6 & 7 & 8 & 9 & 10 & 11 & 12 & 13 & 14 & 15 & 16 & 17 & 18 & 19 & 20 \\
\hline 1 & 48 & 76 & 69 & 76 & 68 & 58 & 57 & 69 & 56 & 70 & 56 & 104 & 83 & 84 & 73 & 62 & 48 & 96 & 65 & 68 \\
\hline 2 & 80 & 119 & 77 & 145 & 68 & 98 & 135 & 183 & 120 & 63 & 163 & 166 & 121 & 164 & 178 & 104 & 159 & 114 & 141 & 119 \\
\hline 3 & 113 & 59 & 104 & 63 & 100 & 143 & 106 & 81 & 104 & 158 & 158 & 28 & 112 & 52 & 85 & 120 & 185 & 117 & 159 & 69 \\
\hline 4 & 90 & 57 & 123 & 68 & 117 & 69 & 67 & 76 & 76 & 101 & 109 & 135 & 58 & 73 & 77 & 82 & 111 & 43 & 89 & 54 \\
\hline 5 & 47 & 66 & 99 & 91 & 55 & 92 & 69 & 88 & 73 & 76 & 96 & 80 & 72 & 74 & 51 & 70 & 87 & 50 & 104 & 81 \\
\hline 6 & 101 & 58 & 95 & 109 & 28 & 78 & 67 & 107 & 71 & 37 & 46 & 64 & 66 & 34 & 30 & 79 & 102 & 100 & 40 & 51 \\
\hline 7 & 103 & 61 & 91 & 70 & 101 & 74 & 77 & 98 & 106 & 80 & 108 & 80 & 64 & 48 & 75 & 69 & 44 & 87 & 113 & 72 \\
\hline 8 & 211 & 192 & 199 & 191 & 113 & 136 & 113 & 125 & 183 & 100 & 118 & 118 & 113 & 194 & 142 & 117 & 140 & 91 & 97 & 148 \\
\hline 9 & 39 & 41 & 61 & 61 & 34 & 69 & 58 & 55 & 47 & 28 & 52 & 27 & 79 & 47 & 39 & 94 & 67 & 56 & 48 & 70 \\
\hline 10 & 98 & 149 & 76 & 105 & 153 & 133 & 43 & 114 & 96 & 220 & 83 & 94 & 99 & 127 & 113 & 23 & 90 & 86 & 113 & 74 \\
\hline 11 & 72 & 108 & 146 & 222 & 146 & 126 & 204 & 142 & 187 & 226 & 147 & 220 & 115 & 227 & 103 & 200 & 206 & 164 & 208 & 153 \\
\hline 12 & 117 & 136 & 108 & 149 & 97 & 126 & 87 & 119 & 93 & 161 & 128 & 113 & 45 & 102 & 150 & 56 & 132 & 89 & 105 & 81 \\
\hline 13 & 101 & 140 & 146 & 207 & 80 & 121 & 157 & 106 & 164 & 152 & 187 & 123 & 108 & 133 & 79 & 192 & 198 & 191 & 152 & 79 \\
\hline 14 & 108 & 174 & 125 & 118 & 130 & 113 & 188 & 121 & 125 & 156 & 156 & 199 & 152 & 91 & 95 & 124 & 139 & 166 & 118 & 132 \\
\hline 15 & 79 & 76 & 67 & 50 & 67 & 61 & 72 & 52 & 36 & 58 & 29 & 53 & 55 & 12 & 63 & 51 & 60 & 44 & 36 & 46 \\
\hline 16 & 102 & 156 & 112 & 137 & 97 & 131 & 64 & 118 & 152 & 46 & 83 & 109 & 92 & 139 & 103 & 143 & 168 & 153 & 42 & 96 \\
\hline 17 & 72 & 37 & 91 & 47 & 92 & 75 & 81 & 46 & 75 & 78 & 49 & 53 & 57 & 97 & 79 & 57 & 56 & 54 & 75 & 43 \\
\hline 18 & 66 & 84 & 85 & 75 & 31 & 78 & 75 & 81 & 57 & 89 & 82 & 58 & 90 & 100 & 59 & 91 & 53 & 109 & 88 & 37 \\
\hline 19 & 133 & 89 & 150 & 134 & 182 & 123 & 131 & 75 & 110 & 140 & 129 & 114 & 134 & 156 & 108 & 192 & 115 & 238 & 148 & 108 \\
\hline 20 & 78 & 87 & 57 & 51 & 119 & 94 & 108 & 99 & 129 & 84 & 23 & 160 & 136 & 108 & 94 & 118 & 74 & 107 & 101 & 69 \\
\hline
\end{tabular}

and $T$ columns were taken from Tables 12 and 13, respectively.

The holding cost $h c_{k}$ and the processing times $t b_{k}$ are all assumed to be 1 for all products $k$. The overtime cost oc is equal to 100 for one unit of overtime. The setup cost $s c_{k}$ is derived based from the average expected demand $\overline{E\left[D_{k}\right]}$ of product $k$

$$
\overline{E\left[D_{k}\right]}=\frac{\sum_{t=1}^{T} E\left[D_{k t}\right]}{T}
$$

and the time-between-orders $T B O$

$$
s c_{k}=\frac{\overline{E\left[D_{k}\right]} \cdot T B O^{2} \cdot h c_{k}}{2} \quad \forall k .
$$

from the solution of the standard Economic Order Quantity model.

The setup time $t s_{k}$ of product $k$ depends on the given parameter $t s^{r e l}$ and the average expected processing time $\overline{E\left[D_{k}\right]} \cdot t b_{k}$ for the average period demand of product $k$

$$
t s_{k}=t s^{r e l} \cdot \overline{E\left[D_{k}\right]} \cdot t b_{k} \quad \forall k \text {. }
$$


To create dynamic capacity parameters $b_{t}$, we divided the expected capacity requirement for processing by the utilization Util due to processing:

$$
b_{t}=\frac{\sum_{k=1}^{K} t b_{k} \cdot E\left[D_{k t}\right]}{U t i l} \quad \forall t .
$$

Note that for instances with setup times, the actual utilization can be substantially higher, in particular in cases with low TBOs and hence many setups.

\section{References}

Absi, N. and S. Kedad-Sidhoum (2009). The multi-item capacitated lot-sizing problem with safety stocks and demand shortage costs. Computers $\&$ Operations Research 36(11), 29262936.

Bihlmaier, R., A. Koberstein, and R. Obst (2009). Modeling and optimizing of strategic and tactical production planning in the automotive industry under uncertainty. OR Spectrum 31(2), 311-336.

Bookbinder, J. H. and J. Y. Tan (1988). Strategies for the probabilistic lot-sizing problem with service-level constraints. Management Science 34(9), 1096-1108.

Brandimarte, P. (2006). Multi-item capacitated lot-sizing with demand uncertainty. International Journal of Production Research 44(15), 2997-3022.

Buschkühl, L., F. Sahling, S. Helber, and H. Tempelmeier (2010). Dynamic capacitated lotsizing problems: a classification and review of solution approaches. OR Spectrum 32(2), $231-261$.

Di Summa, M. and L. A. Wolsey (2008). Lot-sizing on a tree. Operations Research Letters 36, $7-13$.

Fleischmann, B. (2003). Bestandsmanagement zwischen Zero Stock und Inventory Control. OR News (19), 22-27.

Florian, M., J. K. Lenstra, and A. H. G. R. Kan (1980). Deterministic production planning: Algorithms and complexity. Management Science 26, 669-679.

Freimer, M. B., J. T. Linderoth, and D. J. Thomas (2010). The impact of sampling methods on bias and variance in stochastic linear programs. Computational Optimization and Applications, DOI 10.1007/s10589-010-9322-x.

Guan, Y., S. Ahmed, G. L. Nemhauser, and A. J. Miller (2006). A branch-and-cut algorithm for the stochastic uncapacitated lot-sizing problem. Mathematical Programming, Ser. A 105, $55-84$.

Guan, Y. and A. J. Miller (2008). Polynomial-time algorithms for stochastic uncapacitated lot-sizing problems. Operations Research 56(5), 1172-1183.

Helber, S. and K. Henken (2010). Profit-oriented shift scheduling of inbound contact centers with skills-based routing, impatient customers, and retrials. OR Spectrum 32(1), 109-134.

Helber, S. and F. Sahling (2010). A fix-and-optimize approach for the multi-level capacitated lot sizing problem. International Journal of Production Economics 123(2), 247-256.

Jans, R. and Z. Degraeve (2008). Modeling industrial lot sizing problems: a review. International Journal of Production Research 46(6), 1619-1643.

Kanet, J. J., M. F. Gorman, and M. Stoesslein (2010). Dynamic planned safety stocks in supply networks. International Journal of Production Research 48(22), 6859-6880.

Karimi, B., S. M. T. Fatemi Ghomi, and J. M. Wilson (2003). The capacitated lot sizing problem: a review of models and algorithms. Omega 31(5), 365-378. 
Maes, J. and L. N. van Wassenhove (1986). A simple heuristic for the multi item single level capacitated lotsizing problem. Operations Research Letters 4 (6), 265-273.

Martel, A., M. Diaby, and F. Boctor (1995). Multiple items procurement under stochastic nonstationary demands. European Journal of Operational Research 87(1), 74-92.

Mißler-Behr, M. (1993). Methoden der Szenario-Analyse. Wiesbaden: Deutscher Universitätsverlag.

Pochet, Y. and L. A. Wolsey (2006). Production planning by mixed integer programming. New York: Springer.

Robinson, P., A. Narayanan, and F. Sahin (2009). Coordinated deterministic dynamic demand lot-sizing problem: A review of models and algorithms. Omega 37(1), 3-15.

Sahling, F. (2010). Mehrstufige Losgrößenplanung bei Kapazitätsrestriktionen. Gabler Research: Produktion und Logistik. Wiesbaden: Gabler.

Sahling, F., L. Buschkühl, H. Tempelmeier, and S. Helber (2009). Solving a multi-level capacitated lot sizing problem with multi-period setup carry-over via a fix-and-optimize heuristic. Computers \& Operations Research 36(9), 2546-2553.

Saliby, E. (1990). Descriptive sampling: A better approach to monte carlo simulation. Journal of the Operational Research Society 41, 1133-1142.

Sox, C. R., P. L. Jackson, A. Bowman, and J. A. Muckstadt (1999). A review of the stochastic lot scheduling problem. International Journal of Production Economics 62(3), 181-200.

Sox, C. R. and J. A. Muckstadt (1997). Optimization-based planning for the stochastic lotscheduling problem. IIE Transactions 29(5), 349-357.

Tempelmeier, H. (2006). Inventory management in supply networks: Problems, models, solutions. Norderstedt: Books on Demand.

Tempelmeier, H. (2011). A column generation heuristic for dynamic capacitated lot sizing with random demand under a fill rate constraint. Omega 39(6), 627-633.

Tempelmeier, H. and S. Herpers (2010). $\mathrm{ABC}_{\beta}$ - a heuristic for dynamic capacitated lot sizing with random demand under a fillrate constraint. International Journal of Production Research 48(17), 5181-5193.

Tunc, H., O. A. Kilic, S. A. Tarim, and B. Eksioglu (2011). The cost of using stationary inventory policies when demand is non-stationary. Omega 39(4), 410-415.

Winands, E. M. M., I. J. B. F. Adan, and G. J. van Houtum (2011). The stochastic economic lot scheduling problem: A survey. European Journal of Operational Research 210, 1-9.

Zhao, X., J. Xie, and Q. Jiang (2001). Lot-sizing rule and freezing the master production schedule under capacity constraint and determinstic demand. Production and Operations Management 10(1), 45-67.

Zipkin, P. H. (2000). Foundations of inventory management. Boston: McGraw-Hill. 